\title{
Proteomic analysis of early-stage incompatible and compatible interactions between grapevine and P. viticola
}

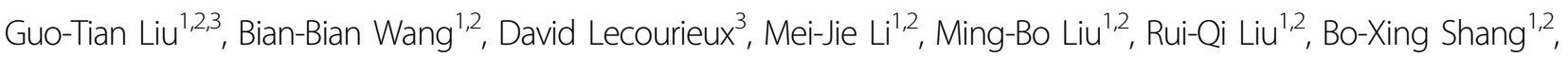
Xiao Yin ${ }^{1,2}$, Li-Jun Wang ${ }^{4}$, Fatma Lecourieux ${ }^{3}$ and Yan $X u^{1,2}$

\begin{abstract}
Wild grapevines can show strong resistance to the downy mildew pathogen $P$. viticola, but the associated mechanisms are poorly described, especially at early stages of infection. Here, we performed comparative proteomic analyses of grapevine leaves from the resistant genotype $V$. davidii "LiuBa-8" (LB) and susceptible V. vinifera "Pinot Noir" (PN) $12 \mathrm{~h}$ after inoculation with P. viticola. By employing the iTRAQ technique, a total of 444 and 349 differentially expressed proteins (DEPs) were identified in LB and PN, respectively. The majority of these DEPs were related to photosynthesis, respiration, cell wall modification, protein metabolism, stress, and redox homeostasis. Compared with PN, LB showed fewer downregulated proteins associated with photosynthesis and more upregulated proteins associated with metabolism. At least a subset of PR proteins (PR10.2 and PR10.3) was upregulated upon inoculation in both genotypes, whereas HSP (HSP70.2 and HSP90.6) and cell wall-related XTH and BXL1 proteins were specifically upregulated in LB and $\mathrm{PN}$, respectively. In the incompatible interaction, $\mathrm{ROS}$ signaling was evident by the accumulation of $\mathrm{H}_{2} \mathrm{O}_{2}$, and multiple APX and GST proteins were upregulated. These DEPs may play crucial roles in the grapevine response to downy mildew. Our results provide new insights into molecular events associated with downy mildew resistance in grapevine, which may be exploited to develop novel protection strategies against this disease.
\end{abstract}

\section{Introduction}

Grapevines (Vitis) include some of the most widely cultivated and most economically important fruit crops worldwide. Grapevine downy mildew is one of the most devastating oomycete diseases of grapevine. Its causal agent, Plasmopara viticola (Berk and Curt) Berl and de Toni, is a strictly obligate biotrophic pathogen that obtains nutrients from living cells of hosts to complete its life cycle through specialized structures called haustoria.

\footnotetext{
Correspondence: Fatma Lecourieux (fatma.ouaked-lecourieux@inrae.fr) or YanXu (yan.xu@nwafu.edu.cn)

'State Key Laboratory of Crop Stress Biology for Arid Areas, College of Horticulture, Northwest A\&F University, Yangling, China

${ }^{2}$ Key Laboratory of Horticultural Plant Biology and Germplasm Innovation in Northwest China, Ministry of Agriculture, Northwest A\&F University, Yangling, China

Full list of author information is available at the end of the article These authors contributed equally: Guo-Tian Liu, Bian-Bian Wang
}

It can infect leaves, shoots, tendrils, inflorescences, and young berries and spread into mature berries through rachis infection.

Plants have evolved sophisticated surveillance systems to defend against pathogen attack ${ }^{1,2}$. These may include preformed constitutive barriers such as a strong cell wall, a thickened waxy layer and dense trichomes on the epidermis, and the presence of antimicrobial toxins in the cell vacuole. In addition, plants are able to activate molecular defense pathways upon contact with pathogens. Such induced defense reactions can provoke pathogenassociated molecular patterns (PAMPs) leading to PAMPtriggered immunity (PTI). However, some pathogens may counter this defense by releasing specific effector molecules that suppress PTI. During the ongoing evolutionary contest between host and pathogen, plants have developed resistance $(\mathrm{R})$ genes encoding receptors that can

\section{() The Author(s) 2021}

(c) (i) Open Access This article is licensed under a Creative Commons Attribution 4.0 International License, which permits use, sharing, adaptation, distribution and reproduction cc) in any medium or format, as long as you give appropriate credit to the original author(s) and the source, provide a link to the Creative Commons license, and indicate if changes were made. The images or other third party material in this article are included in the article's Creative Commons license, unless indicated otherwise in a credit line to the material. If material is not included in the article's Creative Commons license and your intended use is not permitted by statutory regulation or exceeds the permitted use, you will need to obtain permission directly from the copyright holder. To view a copy of this license, visit http://creativecommons.org/licenses/by/4.0/. 
recognize and bind these effectors. This specific recognition triggers a cascade of defense reactions called effector triggered immunity (ETI) ${ }^{3,4}$. This incompatible interaction between pathogens and hosts limits or halts infection. The absence of an effective $\mathrm{R}$ gene product (defined as compatible infection) results in successful infection and colonization.

Plants protect themselves against biotic and abiotic challenges by a diverse array of defense and stress responses. These responses comprise both very rapid changes in gene expression to quickly adapt to the challenges and sustained transcriptional responses to cope with prolonged stress. Although both early and late transcriptional responses are required for optimal defense, early response genes hold the key for perceiving and amplifying the different stress signals and inducing downstream gene expression ${ }^{5-7}$. For example, Li et al. ${ }^{6}$ showed that 37R genes and many genes involved in defense signaling were induced at the early stage of infection (12 hpi). These included genes encoding MAPKs, genes involved in ROS/NO and hormone signaling pathways and genes associated with the synthesis of defenserelated metabolites, such as phenylpropanoids/stilbenoids/ flavonoids ${ }^{6}$. These data highlight the importance of focusing on the characterization of the early mechanisms deployed by grapevine to respond to downy mildew.

Early studies investigating the mechanisms of grapevine resistance to $P$. viticola examined histological and ultrastructural aspects, including callose deposition in stomata, lignification, stilbenic phytoalexin production, hydrogen peroxide $\left(\mathrm{H}_{2} \mathrm{O}_{2}\right)$ accumulation, and hypersensitive reactions $(\mathrm{HRs})^{8-10}$. More recent studies have utilized sequencing technologies to address molecular genetic aspects, leading to greatly enhanced understanding ${ }^{5,6,11-16}$. Figueiredo et al. ${ }^{11}$ identified differences in gene expression and metabolite profiles between resistant and susceptible grapevine cultivars using a combination of cDNA microarray and nuclear magnetic resonance spectroscopy. Wu et al. ${ }^{12}$ characterized gene expression in response to $P$. viticola infection in Vitis amurensis using Solexa sequencing technology, and showed that the differentially expressed genes were mostly associated with ribosome structure, photosynthesis, and amino acid and sugar metabolism. Similarly, to identify genes and pathways associated with downy mildew resistance, $\mathrm{Li}$ et al. ${ }^{6}$ used RNA-based sequencing (RNA-seq) to identify transcriptional responses to infection in a resistant genotype ${ }^{6}$.

Although transcriptional profiling studies have provided new insights into the grapevine response to downy mildew, mRNA abundance does not always reflect the expression level of the respective protein product(s). Protein levels can be influenced by various factors, including the rate of translation and stability of the protein. Proteomic techniques that encompass the extraction and purification of proteins, cleavage to peptides, and detection by mass spectrometry have been well established ${ }^{17}$. In recent years, proteomic-based approaches have been used to study the grapevine response to downy mildew ${ }^{18-23}$. Using two-dimensional gel electrophoresis (2-DE), Milli et al. ${ }^{20}$ identified 82 differentially expressed proteins in grapevine leaves 24, 48 , and $96 \mathrm{~h}$ after inoculation with $P$. viticola. Xu et al. (2015) identified nine proteins expressed at different levels between a susceptible ( $V$. amurensis "Shuangyou") and resistant ( $V$. amurensis "Shuanghong") grapevine genotype after $P$. viticola inoculation using 2-DE followed by MALDI-TOF/TOF ${ }^{18}$. These 2-DE studies have limitations and are more suitable for the identification of abundant proteins, as they rely on the separation and staining of proteins directly in a gel. A more recent proteomic approach, designated iTRAQ (isobaric tags for relative and absolute quantitation), overcomes some limitations of 2-DE-based techniques. iTRAQ is highly sensitive and allows for the identification and quantitation of upto eight samples simultaneously ${ }^{24}$.

Vitis species and cultivars vary in resistance to $P$. viticola. The Chinese wild grapevine $V$. davidii "LiuBa- 8 " (LB) is highly resistant to $P$. viticola, while the $V$. vinifera cultivar "Pinot Noir" (PN) is relatively susceptible ${ }^{25,26}$. In the present study, we used the iTRAQ approach to quantify and assess differences in the proteomes between $\mathrm{LB}$ and $\mathrm{PN}$ at an early stage in response to $P$. viticola. We aimed to gain insight into the early molecular events and to identify candidate proteins involved incompatible and compatible interactions. These proteins could be exploited as markers to develop strategies to protect grapevines against downy mildew.

\section{Results \\ Colonization in LB and PN at 12 hpi}

To evaluate the ability of $P$. viticola to colonize LB and $\mathrm{PN}$, detached leaves from both genotypes were inoculated with cultures of $P$. viticola isolate "YL", and then observed for $12 \mathrm{~h}$ postinoculation (hpi) by aniline blue staining and epifluorescence microscopy. In both LB and PN, zoospores were observed near stomata, and germ tubes, primary hyphae and the first haustorium could be detected (Fig. 1). These observations are consistent with previous reports showing that $P$. viticola can successfully infect and colonize both resistant and susceptible grapevine genotypes $^{5,27,28}$, and suggest that resistance to downy mildew in the LB-resistant genotype involves mechanisms, that are deployed after infection and colonization rather than constitutive physical and chemical barriers.

\section{$\mathrm{H}_{2} \mathrm{O}_{2}$ production in $L B$ and $\mathrm{PN}$ at $12 \mathrm{hpi}$}

To determine whether postinfection resistance mechanisms in $\mathrm{LB}$ involve the production of $\mathrm{H}_{2} \mathrm{O}_{2}$, leaves 

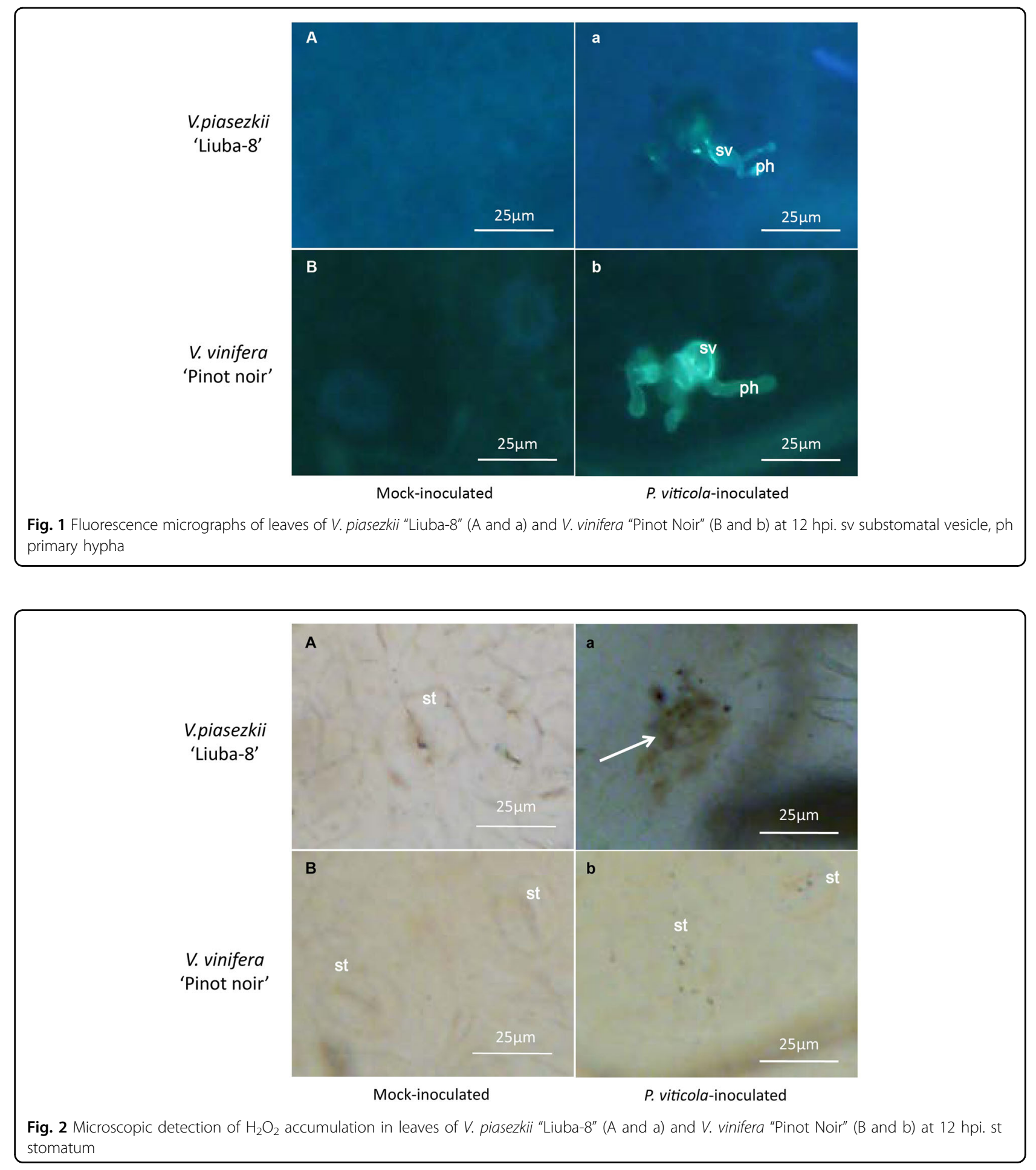

of LB and PN were observed 12 hpi by 3,3-diaminobenzidine $(\mathrm{DAB})$ staining and light microscopy. $\mathrm{DAB}$ reacts with $\mathrm{H}_{2} \mathrm{O}_{2}$ to form an easily visible, reddish-brown precipitate. As shown in Fig. 2, reddish-brown deposits were detectable in LB leaves, while no staining was observed in PN leaves. This result revealed that $P$. viticola infection induced $\mathrm{H}_{2} \mathrm{O}_{2}$ production within 12 hpi in $\mathrm{LB}$ leaves but not in $\mathrm{PN}$ leaves.

\section{Overview of the proteomic analysis}

To compare the diversity and abundance of cellular proteins that accumulate during incompatible and compatible 


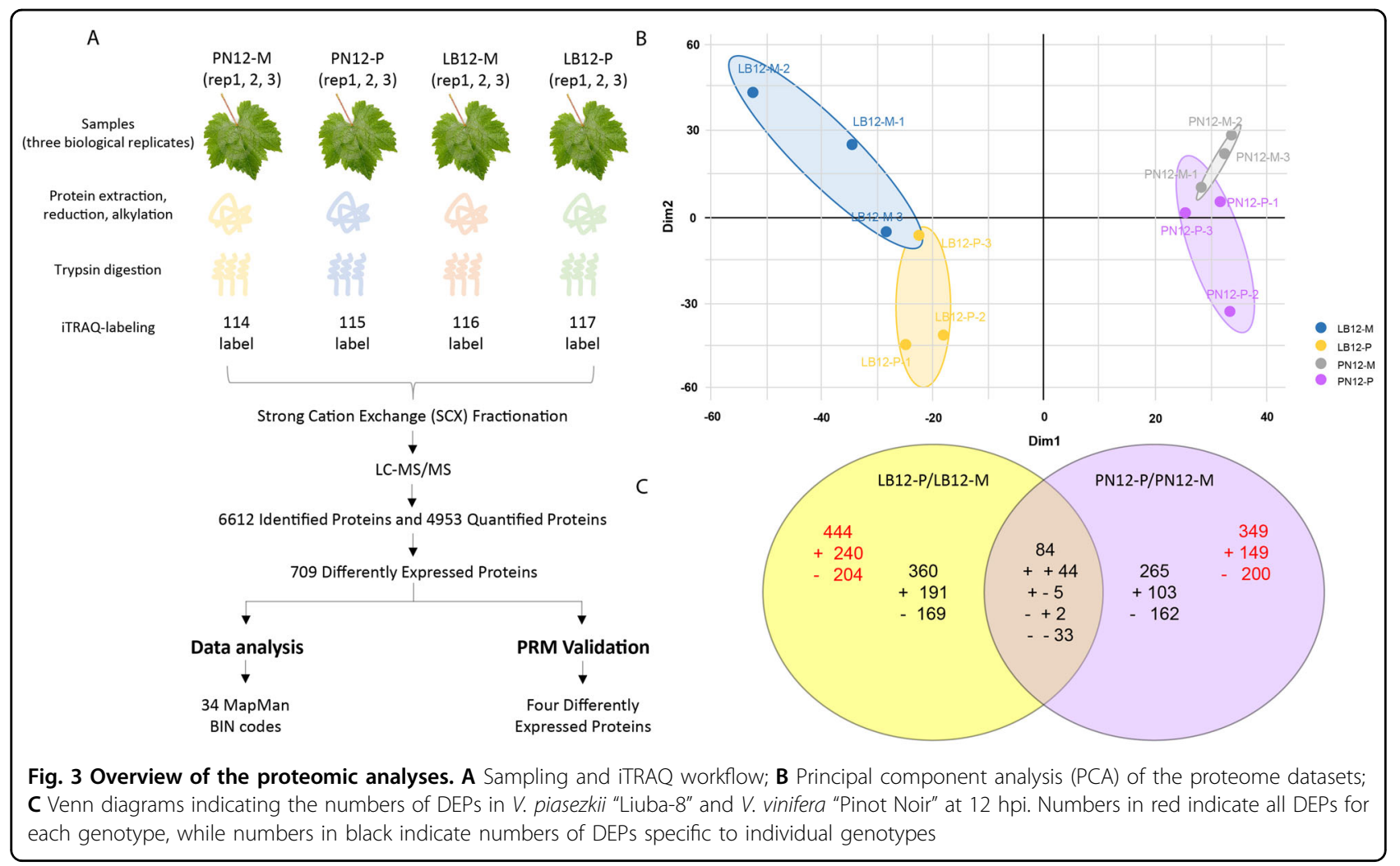

interactions between grapevine and $P$. viticola at an early stage, total proteins were extracted at $12 \mathrm{hpi}$ from $P$. viticola-inoculated $(\mathrm{P})$ or mock-inoculated $(\mathrm{M})$ grapevine leaves (LB12-P, LB12-M, PN12-P, PN12-M, respectively) and analyzed by iTRAQ. The workflow of the analysis is shown in Fig. 3A. In total, 296,872 spectra were obtained, representing 40,165 peptides, 29,605 unique peptides and 6612 proteins. The entire dataset is accessible through ProteomeXchange (PXD018845). Principal component analysis (PCA) of the normalized protein expression data set showed that the three replicates of each experimental condition were well grouped, indicating a high degree of correlation among the replicates (Fig. 3B). Differentially expressed proteins (DEPs) were then identified based on a quantification ratio $>1.2$ or $<0.83$ at $p$ value $<0.05$ and with at least one unique peptide in at least two biological replicates. A total of 709 DEPs were identified in the two genotypes. Furthermore, more DEPs were observed in the resistant genotype LB (444 DEPs) than in the susceptible genotype PN (349 DEPs) (Table S1). In addition, there were more upregulated proteins than downregulated proteins in LB, while in PN, the opposite was observed. Specifically, 240 and 149 proteins were upregulated in $\mathrm{LB}$ and $\mathrm{PN}$, respectively, while 204 and 200 proteins were downregulated in LB and PN, respectively (Fig. 3C). A total of 44 proteins were commonly upregulated in LB and $\mathrm{PN}$, whereas 191 and
103 proteins were specifically upregulated in LB and PN. Among the 84 common proteins, 33 proteins were downregulated in both LB and PN, whereas 169 and 162 proteins were specifically downregulated in LB and PN, respectively. Moreover, five proteins were upregulated in LB but downregulated in $\mathrm{PN}$, while two proteins were downregulated in LB but upregulated in PN (Fig. 3C).

\section{Functional annotation and classification}

Among the 709 DEPs, 538 DEPs were annotated as hypothetical or unknown proteins in UniProt (http:// www.uniprot.org/). To gain more information about these proteins, BLAST (http://www.ncbi.nlm.nih.gov/BLAST/) was used to identify their homologous proteins in the NCBI nonredundant (nr) protein database. In addition, to gain a more detailed description, MapMan was used to conduct the functional annotation and classification of the $\mathrm{DEPs}^{29}$. These 538 DEPs were classified into 34 functional MapMan bins, as shown in Fig. 4. In both LB and PN, the predominant proteins that showed alterations in abundance in response to downy mildew were assigned to photosynthesis, metabolism, stress, and redox categories.

\section{Validation of DEPs by parallel reaction monitoring (PRM)}

PRM technology is an ion monitoring technique based on high-resolution and high-precision mass spectrometry. PRM initially uses the selective detection capability of a 


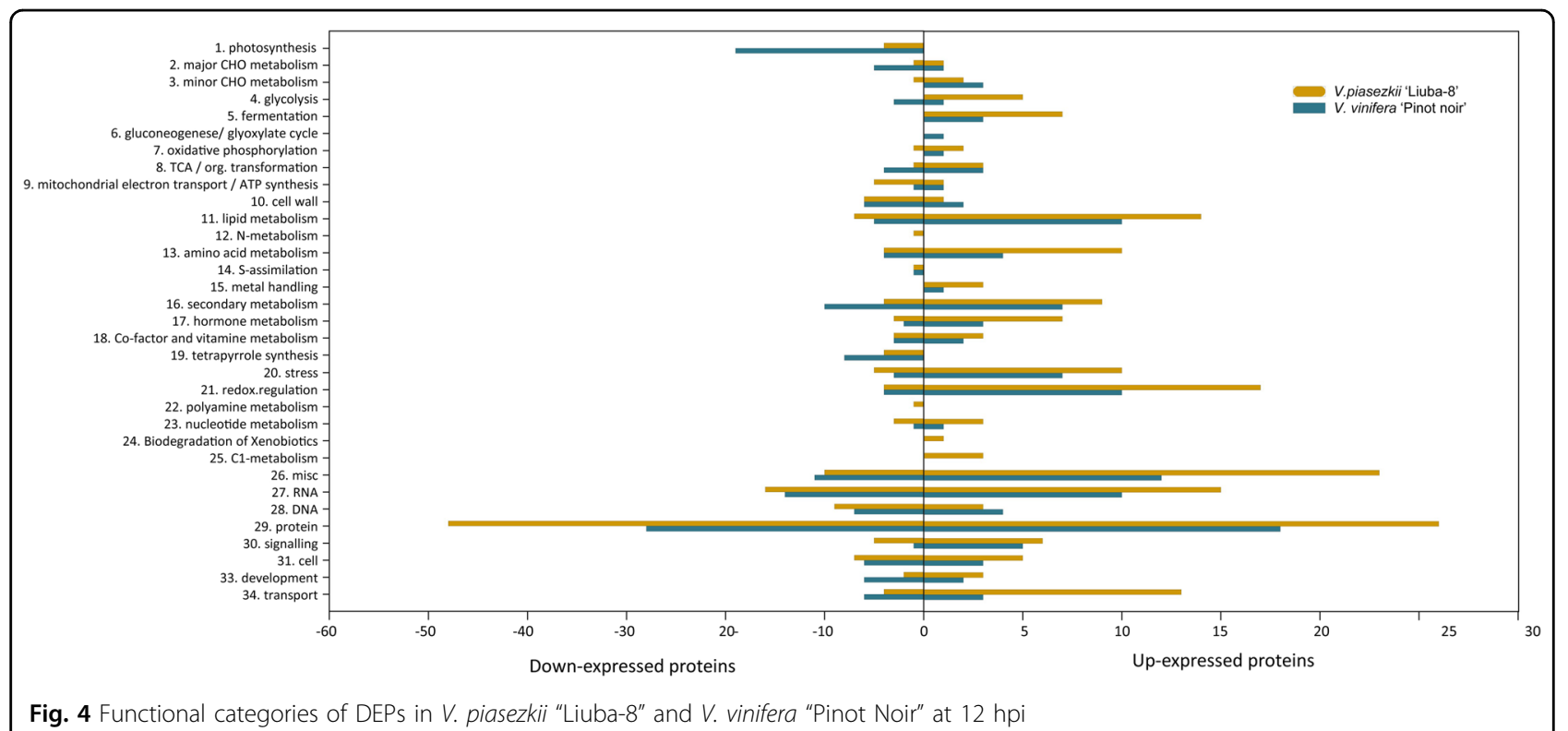

Fig. 4 Functional categories of DEPs in V. piasezkii "Liuba-8" and V. vinifera "Pinot Noir" at 12 hpi

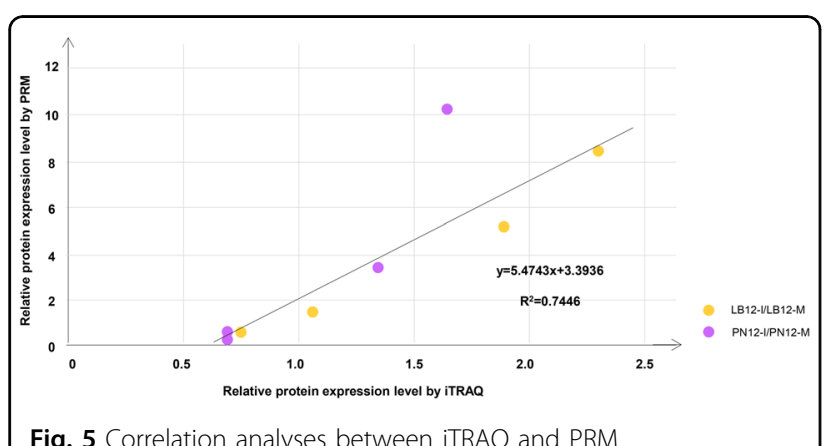

Fig. 5 Correlation analyses between iTRAQ and PRM

quadrupole mass analyzer to selectively detect the precursor ion information of the target peptide. Selected ion precursors are then fragmented by HCD in a collision cell and finally analyzed by a high-resolution and high-massaccuracy Orbitrap analyzer. This technology allows for an accurate and specific analysis of target proteins/peptides in complex samples ${ }^{30}$. To validate the iTRAQ results, four differentially expressed proteins were analyzed using PRM in both varieties at 12 hpi. Data are available from ProteomeXchange (PXD018868). The correlation coefficient between the iTRAQ and PRM analyses was 0.74, which illustrates that the iTRAQ results were reliable for further analysis (Fig. 5 and Table S2).

\section{Transient expression of upregulated proteins confers pathogen resistance in Nicotiana benthamiana}

To preliminarily verify the function of DEPs identified by $\mathrm{iTRAQ}$, five candidate proteins were selected for transient expression in $N$. benthamiana. They were pathogenesis-related proteins (PR5, PR10.2, and PR10.3), calreticulin 2 (CRT2) and a $17.8 \mathrm{kDa}$ class I heat shock protein (HSP17.8). The three PR proteins were highly induced in LB and/or PN after P. viticola infection and have been speculated to play pivotal roles in pathogen resistance. In addition, previous studies have shown that plant disease resistance may result from some constitutively highly expressed genes in the resistant genotype. Therefore, in our study, two candidates (CRT2 and HSP17.8), which showed constitutively high-level expression in resistant LB than PN but were hardly modulated in $\mathrm{LB}$ and $\mathrm{PN}$ in response to $P$. viticola, were also selected.

Genes encoding the five proteins were cloned from LB and PN first (one gene could not be cloned in PN). Sequence alignment showed that the identities of the genes in the two different genotypes reached 90\% (Supplementary Fig. S1). The genes were then cloned into pCAMBIA2300, which harbors a GFP expression cassette (Fig. 6A), and transformed into $N$. benthamiana leaves by Agrobacteriummediated plant genetic transformation. A. tumefaciens cells carrying only GFP were infiltrated into the right panel of the leaf as a control, while cells carrying GFP and genes were infiltrated into the left panel of the same leaf. Western blot analysis showed that all the proteins were successfully expressed in $N$. benthamiana (Fig. 6B). Zoospores of Phytophthora capsici were inoculated onto the agroinfiltrated leaves at 2 days of post-infiltration (dpi). The lesions showed a smaller range in the candidate-transformed leaves than in the control (Fig. $6 \mathrm{C}-\mathrm{G}(\mathrm{c}-\mathrm{g})$ ), indicating that overexpression of these candidates in $N$. benthamiana leaves significantly impeded the growth of $P$. capsici, suggesting that these candidates may contribute to disease resistance in plants. 

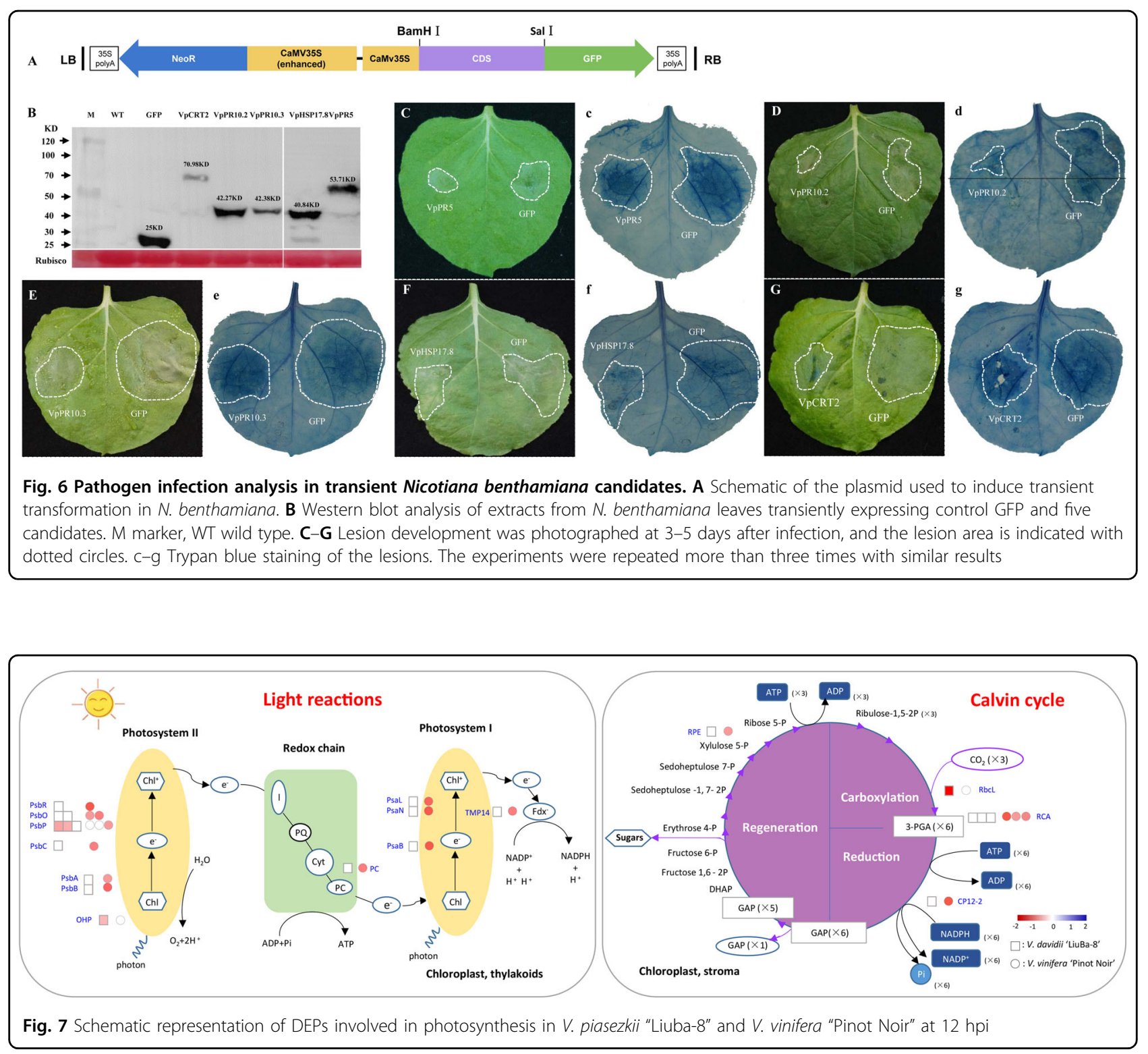

\section{Discussion}

P. viticola is a strictly biotrophic oomycete pathogen that can only survive on living host tissues. Although there have been numerous proteomic studies of plant-pathogen interactions in recent years, few have addressed incompatible and compatible interactions at an early stage of $P$. viticola infection in grapevine, and none have employed iTRAQ. The results of our proteome analysis are discussed below and focus on the various functional groups of proteins.

\section{Photosynthesis inhibition was stronger in the susceptible PN than in the resistant LB}

Photosynthesis is closely associated with plant productivity and energy utilization and is one of the most biotic $^{31}$ and abiotic ${ }^{32,33}$ stress-sensitive physiological processes in plants. Plants are under constant attack by biotic agents such as fungi, bacteria, and viruses, and under these circumstances, the immune system responds quickly to protect against further damage. To be able to withstand this situation, plants allocate more resources from growth to defense, concomitant with a global reduction in photosynthetic capacity. A decrease in photosynthesis has been reported in both incompatible and compatible interactions ${ }^{34-36}$. In this study, 4 and 19 photosynthesis-related DEPs were identified in resistant LB and susceptible PN, respectively, and all of these DEPs were repressed at the early stage irrespective of compatible or incompatible infection (Fig. 7 and Table S3). 
As the primary unit of photosynthesis, PSII is involved in energy absorption, conversion, and photosynthetic electron transport in the light reaction, and its activity and function can be affected by biotic and abiotic stresses. In this study, more than half of the photosynthesis-related proteins identified (3/4 in LB and 8/19 in PN) belonged to PSII, which is consistent with previous studies ${ }^{20,21}$. The PSII core complex comprises PsbA (D1) and PsbB (D2) and the cytochrome b559 subunits a and b. The decrease in abundance of PsbA and PsbB proteins during pathogen infection indicates a decrease in the synthesis/degradation balance of these proteins. This lowered balance in the presence of viral infection results in PSII photoinhibition $^{37,38}$, and consequently a decrease in the reducing power available for carboxylation activity and photorespiration $^{39}$. Kundu et al. ${ }^{40}$ observed a decrease in the abundance of D1 and D2 proteins in $V$. mungo plants infected with Mungbean yellow mosaic indica virus (MYMIV). Moreover, by analyzing photochemical reactions via chlorophyll a fluorescence measurement, they found that very low levels of D1 and D2 were associated with lower actual quantum efficiency of PSII in a susceptible $V$. mungo genotype. These results strongly suggest that MYMIV inhibits PSII, which might limit energy conversion by light reactions ${ }^{40}$. Consistent with these reports, our results showed that D1 and D2 had lower expression in susceptible PN but not in resistant LB. Oxygen-evolving complex (OEC) activity is closely associated with PsbO and PsbP. PsbO is a key structural component of many different types of OECs and functions to stabilize the manganese cluster and modulate the $\mathrm{Ca}^{2+}$ and $\mathrm{Cl}^{-}$requirements for oxygen evolution. $N$. benthamiana plants silenced for PsbO are susceptible to several viruses ${ }^{41}$. In the present study, the abundance of two isoforms of PsbO was decreased in susceptible PN but was not altered in resistant LB, which suggests that PsbO is required for a basal defense mechanism in grapevine P. viticola, as a previous study has reported ${ }^{41}$. PsbP is highly conserved in higher plants and is essential for PSII core assembly and stability. Transient silencing of PsbP in $N$. benthamiana plants enhances pathogenicity and viral DNA accumulation, while overexpression of PsbP impedes disease development during the early phase of infection, suggesting that PsbP participates in the defense response during geminivirus infection ${ }^{42}$. However, PsbP was downregulated in thaumatin-like protein-overexpressing transgenic rice, which exhibited enhanced resistance against bacterial blight ${ }^{43}$. In this study, two PsbP isoforms were slightly decreased in resistant LB, and one PsbP isoform was slightly decreased in susceptible PN. Due to the different functions of PsbP in different plant-pathogen interactions, the specific role of PsbP in the grapevine response to downy mildew needs further investigation. The light-harvesting complex (LHC) is a series of proteins and chlorophyll molecules embedded in the thylakoid membrane in plants, and transfers light energy to a chlorophyll a molecule at the reaction center of the photosystem. However, in our study, only one LHC was found to be downregulated in LB and PN, which may indicate that the LHC is more stable than the PSII core complex in the grapevine response to downy mildew.

Ribulose-1,5-biphosphate carboxylase oxygenase (RubisCO) plays a crucial role in carbon dioxide fixation in the Calvin cycle. RubisCO activase (RCA), which is specifically involved in the activation and maintenance of RubisCO by carbamylation, mediates the defense response to fungi by restoring the catalytic competence of RubisCO by using energy from ATP hydrolysis ${ }^{44}$. It has been reported that genotypes showing a high RCA intensity may have more efficient carbon metabolism and better defenses against pathogens ${ }^{45}$. In contrast, a reduced RCA abundance was reported in compatible plant-virus interactions ${ }^{37}$. Consistent with these reports, our results also showed that the three identified RCA isoforms were all downregulated in PN, but not LB, in response to $P$. viticola.

In general, repression of photosynthesis is an active response to stress perception rather than a secondary physiological response to tissue damage. Once an attack is perceived, plant metabolism must balance potentially competing demands for resources to support defense versus cellular maintenance, growth, and reproduction. In addition, there were fewer repressed proteins in LB than in $\mathrm{PN}$, which indicates that resistance may be associated with a higher recovery capacity. This can also partly explain the smaller effect on the photosynthetic rate observed for cultivars with high resistance than those with low resistance ${ }^{46,47}$. Our findings, together with data from the literature showing significant differences in photosynthetic protein expression between susceptible and resistant grapevine cultivars, suggest that plant responses to biotic stress are not merely physiological but instead are the results of different genetic reprogramming strategies between cultivars.

\section{Glycolysis, the TCA cycle, and the PPP were more induced in resistant $L B$ than in susceptible PN}

Carbohydrate metabolism plays an important role during plant interactions with pathogens. Increases in carbohydrates not only supply massive energy to defense responses, but also to regulate the expression of resistance-related genes.

Glycolysis is a network of reactions with possible sites for substrate movement in and out of various subcellular compartments. Induction of glycolysis in the cytosol facilitates plant acclimation to environmental stress ${ }^{48}$. It has been proposed that the regulation of glycolysis in the leaf sheaths of $R$. solani-infected rice plants is directly 
involved in the regulation of carbon allocation to other pathways, and that this is an important resistance response mechanism $^{48}$. In our study, all glycolysis-related DEPs, including ATP-dependent 6-phosphofructokinase (PFK), glyceraldehyde-3-phosphate dehydrogenase (GAPDH), and pyruvate kinase (PK), were specifically induced in LB (Table 1). In contrast, only one 2-3 biphosphoglycerateindependent phosphoglycerate mutase (PGAM-i) was induced in PN, while two isoforms of $\mathrm{PK}$ and phosphoglucomutase (PGM) were repressed in PN. Increased expression of these enzymes may suggest a strengthened glycolysis pathway, which could lead to generation of ATP and NADPH as a response to pathogen infection. GAPDH catalyzes the reversible conversion of glyceraldehyde 3phosphate to 1,3-bisphosphoglycerate, and thus serves to breakdown glucose to supply energy and carbon for development and abiotic stress and immune responses. Milli et al. ${ }^{20}$ observed the upregulation of two isoforms of GAPDH in the grapevine response to downy mildew, while Figueiredo et al. $^{23}$ showed that GAPDH levels increased in the resistant grapevine genotype "Regent" at $6 \mathrm{hpi}^{23}$. Accordingly, in our study, one isoform of GAPDH was highly expressed in LB but showed no response in $\mathrm{PN}^{20,23}$. However, it has also been reported that GAPDH has functions independent of glycolysis, including mediating ROS signaling ${ }^{49-53}$. Arabidopsis GAPDH knockouts exhibited accelerated programmed cell death (PCD), and an increased electrolyte leakage response to ETI upon inoculation with Pseudomonas syringae ${ }^{51}$. Transient overexpression of cassava cytosolic GAPDH led to decreased resistance against Xanthomonas axonopodis pv manihotis, while its silencing resulted in increased disease resistance ${ }^{52}$. Moreover, the silencing of cytosolic GAPDHs strengthens programmed cell death and resistance in incompatible and compatible interactions ${ }^{50}$. All of these observations indicate that GAPDHs act as negative regulators of plant disease resistance. Whether GAPDH plays a positive role in glycolysis or a negative role in ROS signaling in the grapevine response to downy mildew needs further investigation. PK is a key regulatory enzyme of glycolysis that catalyzes the essentially irreversible stabilization of a phosphate group from phosphoenolpyruvate (PEP) to adenosine diphosphate (ADP), producing one molecule of pyruvate and one of ATP. Capsicum annuum cytosolic pyruvate kinase $1(\mathrm{CaPKc1})$ was induced during the incompatible interaction of hot pepper and tobacco mosaic virus (TMV), indicating that PK could provide pyruvate at a high concentration directly to the mitochondrion, where it might be taken up as a substrate for respiration ${ }^{54}$. PK accumulated at higher levels in the incompatible interaction between $V$. amurensis "Shuanghong" and P. viticola strains "ZJ-1-2" at 12 $\mathrm{hpi}^{6}$. In our study, four PK isoforms were identified in LB and $\mathrm{PN}$ at $12 \mathrm{hpi}$. Two of these were increased in LB, while the other two were decreased in PN. This indicates that when attacked by a pathogen, the plant requires increased ATP released from pyruvate production. The induction of PK in the defense response may be related to the increased energy demands, and therefore, the observed induction of $\mathrm{PK}$ in $\mathrm{LB}$, but not $\mathrm{PN}$, may contribute to resistance in $\mathrm{LB}$ against $P$. viticola.

The tricarboxylic acid (TCA) cycle, which is the second stage of aerobic respiration, is the major energyproducing pathway and generates most of the reduced coenzymes that will be oxidized by the electron transport chain to produce ATP. Carbonic anhydrase (CA) is an enzyme that assists the rapid interconversion of $\mathrm{CO}_{2}$ and $\mathrm{H}_{2} \mathrm{O}$ into carbonic acid, protons and bicarbonate ions. Restrepo et al. reported that silencing CA in N. benthamiana decreased resistance to $P$. infestans ${ }^{55}$, suggesting that $\mathrm{CA}$ is necessary for the resistance response and that pathogens may target this enzyme for suppression in compatible interactions. In our study, one isoform of CA was highly expressed in LB, while the other showed a slight decrease in $\mathrm{PN}$, which supports this hypothesis. Additionally, we identified four and seven DEPs in LB and $\mathrm{PN}$, respectively. In LB, three of the four DEPs were increased, while in $\mathrm{PN}$, four of the seven were decreased. Earlier studies have reported that upregulation of these proteins triggers the TCA cycle to provide additional energy for the defense response through the production of pyruvate and $\mathrm{NADPH}^{40,56}$.

The pentose phosphate pathway (PPP) is the main route for the production of phenolic compounds to activate defense mechanisms. Two enzymes of the PPP, glucose-6-phosphate 1-dehydrogenase (G6PD) and 6phosphogluconate dehydrogenase (6PGD), were induced in LB, while only G6PD was slightly induced in $\mathrm{PN}$. This result is in agreement with previous findings showing that resistant genotypes exhibited high PPP enzyme activities ${ }^{57}$. G6PD and PGD play key roles in the conversion of glucose-6-phosphate to ribulose-5-phosphate, yielding NADPH for reductive biosynthesis and maintenance of the cellular redox state. Pyruvate decarboxylase (PDC) is a homotetrameric enzyme that catalyses the decarboxylation of pyruvic acid to acetaldehyde and carbon dioxide in the cytoplasm of prokaryotes and in the cytoplasm and mitochondria of eukaryotes. Overexpression of PDC in potato conferred a lesion mimic phenotype followed by activation of multiple defense responses leading to significant resistance to $P$. infes$\operatorname{tans}^{58}$. In our study, PDC was strongly increased in LB and only marginally increased in PN. Increased expression of these enzymes suggests a strengthened PPP. The PPP furnishes NADPH to the plasma membrane NADPH oxidase to produce $\mathrm{H}_{2} \mathrm{O}_{2}$, which can act as a signaling molecule for disease resistance. Activation of the PPP also 
Table 1 Respiration-related DEPs in V. piasezkii "Liuba-8" and V. vinifera "Pinot Noir" at 12 hpi

\begin{tabular}{|c|c|c|c|c|c|c|}
\hline Accession & Protein name & Description & $\begin{array}{l}\text { LB12-P/ } \\
\text { LB12-M }\end{array}$ & $p$ value & $\begin{array}{l}\text { PN12-P/ } \\
\text { PN12-M }\end{array}$ & $p$ value \\
\hline D7TBD7 & PFK3 & ATP-dependent 6-phosphofructokinase 3 & 1.3387 & 0.0245 & 1.1625 & 0.0467 \\
\hline D7SXA1 & PFK5 & ATP-dependent 6-phosphofructokinase & 1.6847 & 0.0085 & 1.2523 & 0.1343 \\
\hline F6HG44 & GAPDH & Glyceraldehyde-3-phosphate dehydrogenase & 1.9687 & 0.0011 & 1.2916 & 0.3277 \\
\hline C5DB67 & PGAM-i & $\begin{array}{l}\text { Putative 2-3 biphosphoglycerate independant } \\
\text { phosphoglycerate mutase }\end{array}$ & 1.1062 & 0.2725 & 1.3002 & 0.0186 \\
\hline F6HDW1 & PK & Pyruvate kinase & 0.9113 & 0.2215 & 0.8097 & 0.0462 \\
\hline A5BTB0 & PK & Pyruvate kinase & 1.1932 & 0.0146 & 0.7518 & 0.0290 \\
\hline F6HVY1 & PK & Pyruvate kinase & 2.0420 & 0.0137 & 1.3071 & 0.2677 \\
\hline D7TIZ5 & PK & Pyruvate kinase & 1.2626 & 0.0037 & 0.9238 & 0.1013 \\
\hline D7T1T9 & PGM & Phosphoglucomutase, chloroplastic isoform X2 & 1.0259 & 0.7138 & 0.8204 & 0.0466 \\
\hline D7U830 & PDHA1 & Pyruvate dehydrogenase E1 & 0.9019 & 0.0115 & 0.8121 & 0.0018 \\
\hline F6HI27 & PDHA1 & Pyruvate dehydrogenase E1 component subunit beta-3, chloroplastic & 0.9350 & 0.0434 & 0.7605 & 0.0235 \\
\hline F615U2 & PDHX & $\begin{array}{l}\text { Dihydrolipoamide acetyltransferase component of pyruvate } \\
\text { dehydrogenase complex }\end{array}$ & 1.0167 & 0.8694 & 1.2357 & 0.0174 \\
\hline D7TEL2 & $\mathrm{ACO}$ & Aconitate hydratase & 1.5400 & 0.0002 & 1.2926 & 0.0234 \\
\hline B6VJT4 & $\mathrm{SDH} 3$ & Succinate dehydrogenase subunit 3 & 1.0634 & 0.0046 & 1.3335 & 0.0009 \\
\hline D7SHR6 & CA & Carbonic anhydrase & 1.8017 & 0.0003 & 0.9285 & 0.6448 \\
\hline D7TU30 & CA & Carbonic anhydrase & 0.9075 & 0.5078 & 0.8085 & 0.0138 \\
\hline D7TBH4 & ME & Malic enzyme & 1.4795 & 0.0481 & 0.9893 & 0.9609 \\
\hline D7U0C2 & ME & Malic enzyme & 0.6962 & 0.0332 & 0.6656 & 0.0164 \\
\hline D7UBH2 & G6PD & Glucose-6-phosphate 1-dehydrogenase & 1.2695 & 0.0168 & 1.2169 & 0.0188 \\
\hline $\mathrm{F} 6 \mathrm{HGH} 4$ & 6PGD & 6-phosphogluconate dehydrogenase, decarboxylating & 1.3551 & 0.0027 & 1.1888 & 0.0350 \\
\hline F6HFJ9 & RPI2 & Ribose-5-phosphate isomerase 2 & 0.7215 & 0.0391 & 0.8295 & 0.0695 \\
\hline A5B1B8 & $\mathrm{HACL}$ & 2-hydroxyacyl-CoA lyase & 1.4256 & 0.0116 & 1.0509 & 0.4342 \\
\hline Q43690 & $\mathrm{ADH}$ & Alcohol dehydrogenase 1 & 1.3762 & 0.0001 & 1.0019 & 0.9872 \\
\hline A5COI8 & $\mathrm{ADH}$ & Alcohol dehydrogenase 2 & 1.8392 & 0.0008 & 1.3868 & 0.0380 \\
\hline Q9FZ00 & $\mathrm{ADH}$ & Alcohol dehydrogenase 3 & 1.3686 & 0.0134 & 1.1509 & 0.2686 \\
\hline F6HPN2 & ALDH2B4 & $\begin{array}{l}\text { Aldehyde dehydrogenase family } 2 \text { member B4, mitochondrial } \\
\text { isoform } X_{1}\end{array}$ & 1.3408 & 0.1442 & 1.5281 & 0.0107 \\
\hline D7TCD6 & $\mathrm{ALDH} 2 \mathrm{~A} 1$ & Aldehyde dehydrogenase family 7 member $A 1$ isoform $X 1$ & 1.2873 & 0.0251 & 1.1766 & 0.1921 \\
\hline D7TJI9 & PDC1 & pyruvate decarboxylase 1 & 1.6776 & 0.0007 & 1.2187 & 0.0747 \\
\hline Q9FVE1 & PDC1 & Pyruvate decarboxylase 1 (Fragment) & 1.8848 & 0.0014 & 1.3436 & 0.0487 \\
\hline D7TMQ2 & CS & Citrate synthase & 1.1094 & 0.0600 & 1.4335 & 0.0005 \\
\hline B6VJT7 & Cytb & Cytochrome b & 1.2250 & 0.0491 & 1.0157 & 0.8221 \\
\hline D7TFJ1 & Cytbc1 & Cytochrome b-c1 complex subunit Rieske, mitochondrial & 0.9003 & 0.0126 & 0.8210 & 0.0007 \\
\hline F6HC12 & Cox6b1 & Cytochrome c oxidase subunit $6 \mathrm{~b}-1$ isoform $\mathrm{X} 1$ & 0.8201 & 0.0435 & 0.9918 & 0.9353 \\
\hline $\mathrm{F} 6 \mathrm{HC} 13$ & Cox6b1 & Cytochrome c oxidase subunit $6 \mathrm{~b}-1$ isoform X3 & 0.7543 & 0.0137 & 0.7965 & 0.1050 \\
\hline A5ASQ0 & NDUFA13B & NADH dehydrogenase [ubiquinone] 1 alpha subcomplex subunit 13-B & 0.7919 & 0.0242 & 0.9631 & 0.4828 \\
\hline F6HVC5 & NDUFA6 & NADH dehydrogenase [ubiquinone] 1 alpha subcomplex subunit 6 & 0.7782 & 0.0476 & 1.0859 & 0.4411 \\
\hline
\end{tabular}


Table 1 continued

\begin{tabular}{|c|c|c|c|c|c|c|}
\hline Accession & Protein name & Description & $\begin{array}{l}\text { LB12-P/ } \\
\text { LB12-M }\end{array}$ & $p$ value & $\begin{array}{l}\text { PN12-P/ } \\
\text { PN12-M }\end{array}$ & $p$ value \\
\hline A5AT60 & NDUFB10B & NADH dehydrogenase [ubiquinone] 1 beta subcomplex subunit 10-B & 0.6457 & 0.0239 & 1.0655 & 0.5699 \\
\hline A5BVI0 & $\mathrm{PHB}$ & Prohibitin & 0.9570 & 0.2767 & 1.2479 & 0.0070 \\
\hline
\end{tabular}

Table 2 Cell wall-related DEPs in V. piasezkii "Liuba-8" and V. vinifera "Pinot Noir" at 12 hpi

\begin{tabular}{|c|c|c|c|c|c|c|}
\hline Accession & Protein name & Description & LB12-P/LB12-M & $p$ value & PN12-P/PN12-M & $p$ value \\
\hline EOCRO4 & USPase & UDP-sugar pyrophosphorylase-like & 1.2520 & 0.0032 & 0.9965 & 0.9784 \\
\hline F6l6Y4 & CESA & Cellulose synthase & 0.7945 & 0.0444 & 0.7553 & 0.0810 \\
\hline A5BUX9 & EG & Endoglucanase & 0.7444 & 0.0054 & 0.6423 & 0.0061 \\
\hline F6GW55 & CSLA2 & Glucomannan 4-beta-mannosyltransferase 2 & 0.4878 & 0.0261 & 0.4318 & 0.0376 \\
\hline F6I3Q0 & FAL17 & Fasciclin-like arabinogalactan protein 17 & 0.7666 & 0.0145 & 0.8645 & 0.2261 \\
\hline A5B7N6 & FAL2 & Fasciclin-like arabinogalactan protein 2 & 0.8441 & 0.0153 & 0.7453 & 0.0278 \\
\hline F6GTE2 & UAM1 & UDP-arabinopyranose mutase 1 & 0.9046 & 0.5487 & 0.8219 & 0.0218 \\
\hline $\mathrm{F} 6 \mathrm{H} 740$ & $B X L 1$ & Beta-D-xylosidase isoform X1 & 1.0381 & 0.7833 & 1.8019 & 0.0114 \\
\hline A5AZ70 & $P L$ & Pectate lyase & 0.7407 & 0.0469 & 0.6178 & 0.0387 \\
\hline F614C9 & XTH & Xyloglucan endotransglucosylase/hydrolase 2 & 1.1731 & 0.0722 & 1.7930 & 0.0070 \\
\hline F6HXK9 & PE & Pectinesterase 3 & 0.7891 & 0.0140 & 0.8274 & 0.3513 \\
\hline D7TFE6 & PAE & Pectin acetylesterase 8 & 1.1904 & 0.0841 & 0.7419 & 0.0448 \\
\hline
\end{tabular}

reflects increased demand for precursors of amino and nucleic acid synthesis in infected plants.

Cell wall-related XTH and BXL1 may play a negative role in the grapevine response to downy mildew

Cell wall modification is an important feature of plant adaption to various environmental changes. Structural adjustments of the existing cell wall mediated by cell wallmodifying proteins allow plants to adjust to environmental changes by regulating growth and controlling the entry of biotic agents ${ }^{59}$. Xyloglucan plays a key role in the structure of plant cell walls by cross-linking cellulose microfibrils. Xyloglucan endotransglucosylase/hydrolase (XTH) can decompose xyloglucan chains that are not tightly bound to cellulose and assemble new xyloglucans into the cell walls and is important for the regulation of cell wall strength, extensibility, and tissue integrity ${ }^{60}$. Expression of DkXTH8, a persimmon $\mathrm{XTH}$, in Arabidopsis resulted in increased membrane permeability ${ }^{61}$, while downregulation of XTH NtXET1 in tobacco resulted in the strengthening of cell walls $^{62}$. Polygalacturonase-inhibiting proteins (PGIPs) directly limit the effective ingress of fungal pathogens by inhibiting cell wall-degrading endopolygalacturonases (ePGs). Transgenic tobacco plants expressing grapevine VvPGIP1 exhibited higher resistance to Botrytis infection, which was associated with downregulation of XTH and a decrease in XTH activity (Alexandersson et al. ${ }^{63}$ ). Related to xyloglucan modification, beta-D-xylosidase (BXL1), which is a key enzyme remodeling xylans, was also downregulated. In our study, XTH and BXL1 were highly induced in $\mathrm{PN}$, while no induction was observed in LB (Table 2). These collective results suggest that XTH and BXL1 may play a negative role in the grapevine response to downy mildew. However, this hypothesis needs further investigation.

\section{Protein metabolism was affected in both LB and PN in response to downy mildew}

The MapMan bin "protein" includes amino acid activation, synthesis, targeting, posttranslational modification, degradation, folding, glycosylation, assembly, and cofactor ligation and was the most abundant category in LB (20.8\%) and PN (16.7\%) (Fig. 8 and Table S4). A total of 22 and 5 DEPs were characterized as ribosomal proteins (RPs) in LB and PN, respectively. Although most RPs are thought to be constitutively expressed components of core housekeeping proteins involved in translation, many studies have reported that some RPs may have functions other than ribosome structure and protein biosynthesis, playing a crucial role in the pathogen response. 


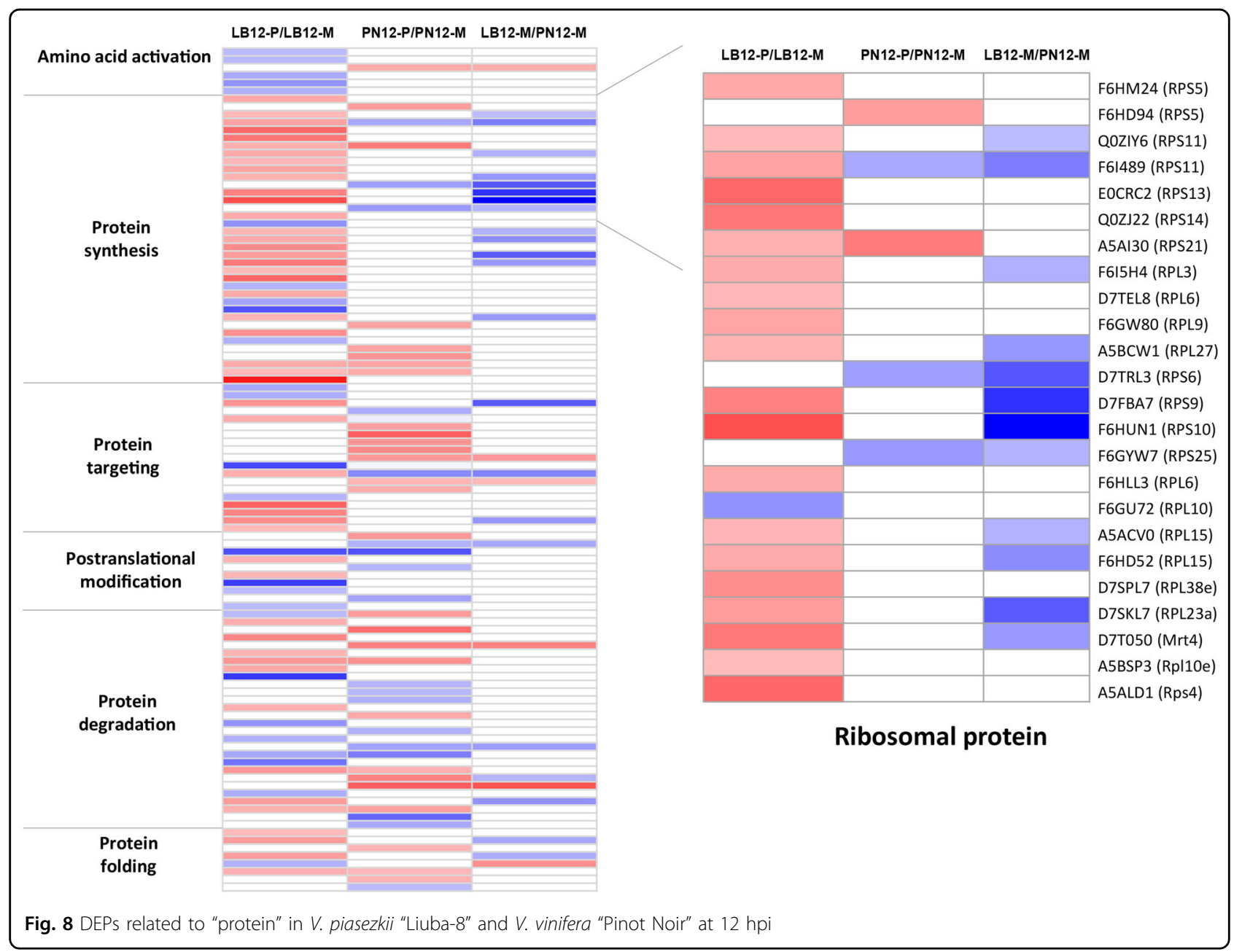

Overexpression of ribosomal protein L13a from eggplant (StoL13a) in $V$. dahliae-sensitive potato conferred enhanced resistance to $V$. dahliae infection, which was associated with a reduction in ROS and attenuated oxidative injury ${ }^{64}$. In Arabidopsis, overexpression of ribosomal protein L18 from cotton (GaRPL18) conferred enhanced resistance to $V$. dahlia infection, while silencing of GaRPL18 increased susceptibility to $V$. dahliae compared with the control by decreasing the abundance of immune-related molecules ${ }^{65}$. However, in our study, all RPs except RPL10 were repressed in LB (Table S4). Similar to our results, 28 of 34 RPs exhibited decreased expression in the resistant cucumber line SSL508-28 ${ }^{66}$. The functions and mechanisms of RPs in stress responses remain largely unknown, and further studies are required to understand the role of differentially expressed RPs during the grapevine response against downy mildew. Previous studies have found that protein phosphatases $2 \mathrm{C}$ (PP2Cs) are also involved in plant-microbe interactions. Transgenic expression of two rice PP2Cs, OsBIPP2C1 and OsBIPP2C2, in tobacco conferred enhanced resistance to tobacco mosaic virus and $P$. parasitica ${ }^{67,68}$. In our study, one PP2C protein was highly induced in LB, while no induction was observed in $\mathrm{PN}$, suggesting a putative role for $\mathrm{PP} 2 \mathrm{C}$ in the disease resistance response.

\section{Stress-related proteins were highly expressed upon infection in both resistant LB and susceptible PN}

Pathogenesis-related (PR) proteins play pivotal roles in the plant response to pathogen challenge. PR proteins are divided into 17 classes, PR1-PR17, based on their amino acid sequence, serological relationship, and enzymatic activities $^{69}$. Four PR proteins (PR4, PR5, PR10.2, and PR10.3) were identified in our study. PR4, which is a chitinase and chitin-binding protein, plays a significant role in pathogen responses in many plants. Transgenic overexpression of PR4 in grapevine conferred increased resistance to powdery mildew, while loss-of-function PR4 mutations increased susceptibility to downy mildew in grapevine $^{70,71}$. In our study, PR4 was induced upon infection in LB but not PN (Table 3). Moreover, even in mock-inoculated plants, PR4 was more abundant in LB 
Table 3 Stress-related DEPs in V. piasezkii "Liuba-8" and V. vinifera "Pinot Noir" at 12 hpi

\begin{tabular}{|c|c|c|c|c|c|c|}
\hline Accession & Protein name & Description & LB12-P/LB12-M & $p$ value & PN12-P/PN12-M & $p$ value \\
\hline A5BS35 & BSP & Basic secretory protease & 1.1686 & 0.1613 & 1.3090 & 0.0003 \\
\hline A5C9R1 & $\mathrm{Bl}-1$ & Bax inhibitor 1-like & 1.2685 & 0.0186 & 1.0341 & 0.5750 \\
\hline D7SI01 & EDS1 & Protein EDS1 & 1.2917 & 0.0346 & 1.2653 & 0.0929 \\
\hline A5BFQ1 & LRRNT_2 & LRRNT_2 domain-containing protein & 0.8078 & 0.0231 & 0.8854 & 0.3139 \\
\hline D7T2C8 & PR4 & Endochitinase $\mathrm{A}$ isoform $\mathrm{X} 1$ & 2.1435 & 0.0144 & 1.0627 & 0.6999 \\
\hline A5ASS2 & PR5 & Thaumatin & 1.8192 & 0.0142 & 1.4767 & 0.1142 \\
\hline Q9FS43 & PR10.2 & Pathogenesis-related protein 10.2 & 2.2944 & 0.0056 & 1.6451 & 0.0145 \\
\hline B7SL50 & PR10.3 & Pathogenesis-related protein 10.3 & 1.5780 & 0.0084 & 1.9446 & 0.0049 \\
\hline F6HXP8 & HSP17.4 & 17.4 kDa class III heat shock protein & 1.2575 & 0.0493 & 0.9549 & 0.6525 \\
\hline A5B2NO & HSP18.2 & 18.2 kDa class I heat shock protein & 1.4104 & 0.1425 & 1.5049 & 0.0255 \\
\hline A5AUN6 & HSP70 & Heat shock $70 \mathrm{kDa}$ protein $1 \mathrm{~A} / 1 \mathrm{~B}$ isoform $\mathrm{X} 5$ & 1.3001 & 0.1353 & 1.3387 & 0.0424 \\
\hline A5AGD9 & $\mathrm{HSC70}$ & Heat shock cognate $70 \mathrm{kDa}$ protein & 1.2647 & 0.0470 & 1.2876 & 0.0274 \\
\hline F6GV26 & HSC70.2 & Heat shock cognate $70 \mathrm{kDa}$ protein 2 & 1.6785 & 0.0342 & 1.2094 & 0.0549 \\
\hline A5AEP7 & HSP90.6 & Heat shock protein $90-6$, mitochondrial isoform $\times 1$ & 1.7312 & 0.0257 & 1.3159 & 0.0709 \\
\hline $\mathrm{F} 6 \mathrm{HOH} 3$ & PMT10 & Methyltransferase PMT10 & 0.7534 & 0.0434 & 0.7963 & 0.0879 \\
\hline F6HTC6 & PMT14 & Methyltransferase PMT14 & 1.1029 & 0.1431 & 0.8011 & 0.0230 \\
\hline F6HPC4 & PMT2 & Methyltransferase PMT2 & 0.8299 & 0.0196 & 0.9919 & 0.9519 \\
\hline F610C7 & PMT26 & Methyltransferase PMT26 & 0.8329 & 0.0279 & 0.7959 & 0.0028 \\
\hline A5BJD9 & MLP423 & MLP-like protein 423 & 1.1341 & 0.1345 & 0.8242 & 0.0355 \\
\hline A5BJA2 & STS14 & STS14 protein & 0.7591 & 0.0490 & 0.9046 & 0.7775 \\
\hline D7U418 & USP & Usp domain-containing protein & 1.1801 & 0.1098 & 1.5095 & 0.0251 \\
\hline
\end{tabular}

than in PN. Based on this observation, we speculate that PR4 has a basal function in pathogen resistance in grapevine. PR5, also called thaumatin, is another wellcharacterized defensive protein in various plants. Transgenic plants expressing thaumatin at high levels exhibited enhanced resistance against various pathogens ${ }^{72-74}$. PR5 is supposed to produce transmembrane pores and inhibit fungal growth by restraining spore germination and germ tube growth ${ }^{75}$. PR5 has been widely reported to be induced in response to $P$. viticola infection in grapevine, but its specific role remains obscure, as contradictory results have been reported. One study found that PR5 was constitutively expressed in both susceptible and resistant grapevine cultivars ${ }^{10}$, whereas another study reported that PR5 was expressed at lower levels during the $P$. viticola incompatible interaction than during the compatible interaction $^{6}$. In our study, PR5 was induced more strongly in the resistant $\mathrm{LB}$ than in the susceptible PN. Moreover, transient overexpression of VpPR5 significantly impeded the growth of $P$. capsici, suggesting that VpPR5 confers pathogen resistance in $N$. benthamiana (Fig. 6C, c). However, the specific function of PR5 needs further investigation in the context of the grapevine response to downy mildew. PR10 is an important protein of the plant response to fungal invasion that displays antimicrobial activities and in vitro ribonuclease (RNase) activities ${ }^{76-79}$. This RNase activity may protect plants during PCD at infection sites or act directly upon pathogens. In grapevine, PR10 proteins or transcripts accumulate in response to various biotic stresses, including $P$. viticola infection $^{5,20,80,81}$. However, only a subset of PR10 gene family members are induced upon pathogen infection in grapevine. He et al. reported that the induction of three PR10 genes (VpPR10.2, $V v P R 10.2$, and $V v P R 10.3)$ in response to $P$. viticola was dependent on a pyrimidine-rich cis-acting element in their promoters ${ }^{79}$. PR10.2 was more strongly expressed in $V$. pseudoreticulata than in $V$. vinifera in response to $P$. viticola infection, thus suggesting that it contributes to the strong downy mildew resistance of $V$. pseudoreticulata. Additionally, transgenic overexpression of VpPR10.2 in a susceptible $V$. vinifera genotype enhanced resistance to $P$. viticola ${ }^{79}$. In our study, both PR10.2 and PR10.3 were strongly induced in LB and $\mathrm{PN}$. In addition, similar to previous research results, 
transgenic overexpression of VpPR10.2 and VvPR10.3 conferred pathogen resistance in $N$. benthamiana (Fig. 6D, E and d, e). These combined results suggest that VpPR10.2 and VpPR10.3 may play pivotal roles in the response of grapevine to $P$. viticola.

In addition to proteins in the PR family, the stressrelated group of DEPs included heat shock proteins (HSPs). HSPs assist in the proper folding of newly synthesized proteins, act in innate immune responses and are essential in inducing other resistance proteins. Based on molecular mass, there are five major HSP subfamiliesHSP100, 90, 70, 60, and small HSP (sHSP)-conservatively recognized as molecular chaperones. Plants respond to pathogen invasion using two distinct innate immune responses mediated by pattern recognition receptors (PRRs) or $\mathrm{R}$ proteins. HSPs play an indispensable role as molecular chaperones in the quality control of plasma membrane-resident PRRs and intracellular $\mathrm{R}$ proteins ${ }^{82}$. We identified four and three $P$. viticola-induced HSPs in $\mathrm{LB}$ and $\mathrm{PN}$, respectively. HSP70 plays a crucial role in the plant response to pathogen infection ${ }^{83-87}$. In our study, half of the differentially expressed HSP70s were induced in both LB and PN. Moreover, HSC70-2 was more abundant in LB than in $\mathrm{PN}$, which is in agreement with a previous study showing that HSC70 is especially abundant in Regent at an early stage of infection $(6 \mathrm{hpi})^{23}$. HSC70 regulates Arabidopsis immune responses ${ }^{88}$ and participates in both positive and negative regulation of PCD and immunity signaling ${ }^{89,90}$. HSP90 functions in protein complexes with a large set of cochaperones. In Arabidopsis, HSP90 interacts with Mla12 resistance (RAR1) and suppressor of the G2 allele of $s k p 1$ (SGT1) to coordinate the RPM1 function in disease resistance ${ }^{91,92}$. In addition, HSP90 is essential for R gene (R3a)-mediated hypersensitivity and suppresses INF1-induced cell death activation by an RxLR effector (AVR3aKI) in the $N$. benthamiana defense against Phytophthora infestans ${ }^{93}$. In our study, the HSP90 protein Hsp90.6 was especially induced in the resistant cultivar LB. Whether Hsp90 functions as a chaperone in grapevine resistance to $P$. viticola needs further investigation. sHSPs are ATP-independent chaperones that especially interact with unfolded proteins to prevent unfolding and subsequent aggregation. sHSPs are commonly associated with abiotic stresses; however, studies have also reported that sHSPs may play critical roles in plant immunity ${ }^{94-97}$. One hypothesis is that chaperone activity can aid the stabilization and accumulation of $\mathrm{R}$ proteins. Van Ooijen et al. reported that a tomato sHSP, named RSI2, confers resistance to Fusarium oxysporum by interacting with the LRR domain of R protein I-2, while silencing RSI2-related sHSPs in $N$. benthaminana compromised the $\mathrm{R}$ protein I-2-mediated $\mathrm{HR}^{97}$. StHSP17.8, which was highly induced in a resistant genotype of potato against late blight infection, can interact with heat shock elements (HSEs) present in the StWRKY1 promotor region to enable the functioning of StWRKY in response to potato against $P$. infestants ${ }^{96}$. In our study, HSP17.8 showed no difference in LB and PN after $P$. viticola infection. However, HSP17.8 had a constitutively higher expression level in LB than in PN. Moreover, transient overexpression of VpHSP17.8 significantly impeded the growth of $P$. capsici (Fig. 6F, f). All the previous findings, together with the results provided in the present study, suggest that VvHSP17.8 is required for pathogen resistance.

Calreticulin (CRT) is a highly conserved calcium-binding molecular chaperone that facilitates the folding of newly synthesized glycoproteins and regulates $\mathrm{Ca}^{2+}$ homeostasis in the endoplasmic reticulum (ER) lumen ${ }^{98,99}$. It has been reported that CRT isoforms (CRT1, CRT2, and CRT3) are important regulators of plant innate immunity. CRT2 appears to have a dual regulatory role in plant defense against biotrophic pathogens ${ }^{100}$. Although overexpression of CRT2 induced SA accumulation and activated some systemic acquired resistance-associated marker genes, it also resulted in increased susceptibility to Pst DC3000. However, in our study, CRT2 showed a higher constitutive expression level in LB than in PN. In addition, in contrast to the aforementioned results, transient overexpression of VpCRT2 increased resistance to $P$. capsici in $N$. benthamiana (Fig. 6G, g). Since there are still relatively few studies of CRT2, future studies will be required to elucidate the role of CRT2 in plant immunity.

\section{Fine-tuning of the redox status in the grapevine response to downy mildew}

When experiencing pathogen invasion, plants deploy various defense mechanisms, including the oxidative burst leading to a rapid production of ROS (especially $\mathrm{H}_{2} \mathrm{O}_{2}$ ). The accumulation of ROS marks the successful recognition of infection and the activation of plant defense responses. ROS play an essential role in pathogen resistance by directly reinforcing cell walls through crosslinking of glycoproteins and lipid peroxidation ${ }^{101}$. Previous studies have shown that $\mathrm{H}_{2} \mathrm{O}_{2}$ accumulates more rapidly in resistant genotypes than in susceptible genotypes in many plant-pathogen interactions ${ }^{23,102,103}$. In grapevine, $\mathrm{H}_{2} \mathrm{O}_{2}$ accumulated more quickly and to higher levels in "Regent" than in a susceptible genotype ${ }^{23} \cdot \mathrm{H}_{2} \mathrm{O}_{2}$ production is one of the earliest (12 hpi) detectable cytological events against downy mildew in the resistant grapevine clutivar "Solaris" ${ }^{104}$. In the present study, $\mathrm{H}_{2} \mathrm{O}_{2}$ accumulated in LB but not in PN by 12 hpi (Fig. 2). These results showed that in $\mathrm{LB}, \mathrm{H}_{2} \mathrm{O}_{2}$ plays an important role in early defense against downy mildew. In general, ROS bursts constitute some of the earliest plant responses to pathogen invasion, and as signaling molecules, ROS can 
regulate PCD during pathogen infection. ROS levels depend on the balance between ROS production and scavenging ${ }^{105}$, and when excess ROS are produced, injuries will occur. ROS amounts depend both on enzymatic and nonenzymatic scavenging molecules such as superoxide dismutase (SOD), ascorbate peroxidase (APX), catalase (CAT), peroxidase (POX), and the antioxidants ascorbate (ASC), glutathione (GSH), and glutathione peroxidases (GPX), which offer a highly efficient system for maintaining ROS homeostasis ${ }^{105,106}$. Antioxidant enzymes are induced against pathogens in both resistant and susceptible genotypes, although at varying levels. In this study, we identified 21 and 14 differentially expressed ROS-associated proteins in $\mathrm{LB}$ and $\mathrm{PN}$, respectively, and most of them (17 in LB and 10 in PN) showed higher expression in inoculated leaves than in the corresponding mock control (Table 4). APX is a key enzyme in the ascorbate-glutathione cycle, an important antioxidant system that is able to detoxify ROS in plant cells. In grapevine, APX has been shown to be induced by pathogen infection ${ }^{20,107-109}$. In our analysis, the higher expression of two APXs in LB relative to PN may contribute to resistance in LB. POX catalyzes the reduction of $\mathrm{H}_{2} \mathrm{O}_{2}$ and a variety of organic and inorganic hydrogen donors. During this process, substrates are oxidized, promoting lignin formation in the cell wall. Many studies have reported the involvement of POXs in pathogen responses ${ }^{110-112}$. These POXs may also promote changes in plant cell walls to form a physical barrier blocking invasion. We found that in $\mathrm{PN}$, four of the seven identified POXs were upregulated, consistent with a role in resistance. Furthermore, we did not identify any upregulated POX in LB, suggesting that genotype-specific resistance in PN may be due at least partly to a strengthened cell wall. Glutathione S-transferases (GSTs) are an isozyme family catalyzing the conjugation of the reduced form of glutathione to a number of electrophilic substrates, such as phytotoxic compounds, for detoxification. GSTs detoxify metabolites or phytotoxins produced during oxidative damage or pathogen infection ${ }^{113,114}$. Previous studies have shown that GSTs contribute to resistance against powdery mildew. In wheat, GSTF5 was more highly expressed during an incompatible interaction than during a compatible interaction ${ }^{115}$. Moreover, in powdery mildewinfected tomato, a GST gene was more rapidly upregulated in a resistant wild genotype harboring the $\mathrm{Ol}-1$ resistance gene than in a susceptible genotype. Virusinduced gene silencing was used to reduce the expression of this GST gene in resistant plants, and the GST-silenced plants showed a susceptible phenotype after inoculation with $O$. neolycopersici ${ }^{116}$. In our study, a total of 11 isoforms of GSTs were identified, and all of these isoforms showed increased expression in both resistant LB and susceptible PN. However, more GST isoforms were detected in resistant LB than in susceptible PN. These results imply that GSTs may be involved in grapevine resistance to downy mildew.

\section{Conclusion}

Grapevine downy mildew is one of the most devastating grapevine oomycete diseases worldwide. Vitis vinifera cultivars are generally susceptible to the downy mildew pathogen $P$. viticola, whereas wild grapevines can show strong resistance. Comparative proteomics of grapevine leaves from the resistant genotype $V$. davidii "LiuBa-8" (LB) and the susceptible genotype $V$. vinifera "Pinot Noir" $(\mathrm{PN})$ at 12 hpi were conducted to understand the complex relationship of incompatible and compatible interactions between grapevine and $P$. viticola at the early stage of infection. A total of 444 and 349 DEPs were identified in $\mathrm{LB}$ and PN, respectively, at $12 \mathrm{hpi}$ by iTRAQ. The majority of these DEPs were related to photosynthesis, respiration, cell wall modification, protein metabolism, stress, and redox homeostasis (Fig. 9). Our broad comparative characterization of resistant and susceptible genotypes provides insights into the molecular events and identifies candidate proteins underlying incompatible and compatible interactions; these resources might be exploited to develop new protection strategies against downy mildew in grapevine.

\section{Materials and methods}

\section{$P$. viticola isolates, plant materials, and sample collection}

$P$. viticola isolate "YL" was selected for its consistent and high production of sporangia. It was originally isolated as a single sporangiophore from a leaf of hybrid grapevine at the Grape Repository of Northwest A\&F University, Yangling, Shaanxi, China, showing typical symptoms of downy mildew according to our previous method $^{26}$. Briefly, P. viticola was serially infected three times by transferring just one sporangiophore from the contaminated leaves. Next, the isolate was reproduced weekly on the separated PN leaves, which were placed in a $90 \mathrm{~mm}$ Petri dish (off-axis surface facing up) on wet filter paper. In addition, the isolate was maintained in a controlled environment with $80 \%$ relative humidity that was bright at $22^{\circ} \mathrm{C}$ for $16 \mathrm{~h}$ and then dark at $18{ }^{\circ} \mathrm{C}$ for $8 \mathrm{~h}$. The third and fourth leaves from the apex of the PN and LB vertices were collected. The surfaces of these leaves were disinfected with $0.01 \%$ bleach, and sterile distilled water (SDW) was then used to rinse the leaves three times. Leaf disks with a diameter of $10 \mathrm{~mm}$ were obtained by using a sanitized cork borer. Leaf abaxial surfaces were inoculated with $50 \mu \mathrm{L}$ drops of an aqueous suspension of $5 \times 10^{4}$ sporangia per $\mathrm{mL}$ and placed on wet filter paper in $90 \mathrm{~mm}$ Petri dishes. The control groups of leaf disks were inoculated with $50 \mu \mathrm{L}$ drops of sterile distilled water. Incubation conditions were as 
Table 4 Redox-related DEPs in V. piasezkii "Liuba-8" and V. vinifera "Pinot Noir" at 12 hpi

\begin{tabular}{|c|c|c|c|c|c|c|}
\hline Accession & Protein name & Description & LB12-P/LB12-M & $p$ value & PN12-P/PN12-M & $p$ value \\
\hline D7SKR5 & APX2 & L-ascorbate peroxidase 2, cytosolic & 1.2619 & 0.0046 & 1.1535 & 0.0765 \\
\hline F61106 & APX3 & L-ascorbate peroxidase 3 , peroxisomal & 1.2350 & 0.0172 & 1.0673 & 0.6705 \\
\hline A5AL13 & GME & GDP-mannose 3,5-epimerase 2 isoform $\mathrm{X} 1$ & 1.2291 & 0.0406 & 1.0084 & 0.8876 \\
\hline A5JPK5 & GME & GDP-mannose-3',5'-epimerase & 1.6526 & 0.0209 & 1.0241 & 0.8189 \\
\hline D7TS92 & $G R$ & Glutathione reductase & 1.3918 & 0.0136 & 1.2166 & 0.0347 \\
\hline F6HLE2 & OXP1 & 5-Oxoprolinase & 1.2065 & 0.0051 & 1.1469 & 0.0234 \\
\hline F6HQJ3 & HB2 & Hemoglobin-2 & 3.1828 & 0.0465 & 1.9652 & 0.1981 \\
\hline D7U252 & GST & Glutathione S-transferase & 1.0140 & 0.9072 & 1.3040 & 0.0286 \\
\hline F6HZU2 & GST & Glutathione S-transferase & 1.5318 & 0.0968 & 1.9754 & 0.0030 \\
\hline D7T7H2 & GST & Glutathione S-transferase & 1.8345 & 0.0154 & 1.3628 & 0.1398 \\
\hline D7TQY1 & GST & Glutathione S-transferase & 1.5083 & 0.0186 & 1.0644 & 0.5396 \\
\hline A5AG54 & GST & Glutathione S-transferase & 1.3174 & 0.0347 & 1.3280 & 0.0878 \\
\hline A5BCR8 & GST & Glutathione S-transferase & 1.4124 & 0.0137 & 1.2549 & 0.0681 \\
\hline D7TQB6 & GSTL3 & Glutathione S-transferase L3 & 1.6252 & 0.0296 & 1.3937 & 0.1597 \\
\hline D7TQB0 & GSTL3 & Glutathione S-transferase L3 & 1.3519 & 0.0339 & 1.2717 & 0.0556 \\
\hline F6H8J1 & GST & Glutathione transferase GST 23 & 1.8629 & 0.0048 & 1.2489 & 0.5001 \\
\hline F6HR78 & GST & Gutathione S-transferase & 1.2371 & 0.0018 & 1.2420 & 0.0411 \\
\hline A5AQA0 & GST & Gutathione S-transferase U7 & 1.1900 & 0.0182 & 1.3175 & 0.0058 \\
\hline A5BPH1 & GHR & Glutathionyl-hydroquinone reductase YqjG & 1.3409 & 0.0125 & 1.2175 & 0.0009 \\
\hline F6HIK4 & POX & Peroxidase & 0.7664 & 0.0006 & 0.9086 & 0.2290 \\
\hline A5C5U0 & POX & Peroxidase & 0.8881 & 0.3360 & 0.7934 & 0.0155 \\
\hline F6GXY7 & POX & Peroxidase & 1.0961 & 0.3932 & 1.4876 & 0.0231 \\
\hline A5B8V0 & POX & Peroxidase & 1.6515 & 0.0739 & 1.4642 & 0.0466 \\
\hline F6GY60 & POX & Peroxidase 12 & 0.9447 & 0.6975 & 1.3850 & 0.0412 \\
\hline A7NY33 & POX & Peroxidase 4 & 1.0229 & 0.8431 & 1.2716 & 0.0262 \\
\hline F6GWS4 & POX & peroxidase 72 & 0.7085 & 0.0389 & 0.8475 & 0.5531 \\
\hline G1JT87 & Prx $\|$ & Peroxiredoxin & 0.8595 & 0.2273 & 0.7557 & 0.0436 \\
\hline A5BAW6 & TRX & Peroxiredoxin-2E, chloroplastic & 0.9841 & 0.8486 & 0.6625 & 0.0120 \\
\hline D7TES2 & SOD & superoxide dismutase $[\mathrm{Fe}] 3$, chloroplastic isoform & 0.6570 & 0.0165 & 0.6750 & 0.0708 \\
\hline E2GMW1 & TRX-H & Thioredoxin $\mathrm{h}$ & 1.1379 & 0.5355 & 0.7204 & 0.0061 \\
\hline A9UFY2 & TRX-H & Thioredoxin $\mathrm{h}$ & 1.2989 & 0.0232 & 1.1893 & 0.2921 \\
\hline D7T9N8 & TO1 & Thioredoxin O2, mitochondrial & 0.8273 & 0.0331 & 1.1203 & 0.0380 \\
\hline
\end{tabular}

described above. Three independent biological replicates were collected for each condition $(P$. viticolainoculated and mock-inoculated), each comprising a pool of three leaves from three different plants.

Visualization of $P$. viticola and localization of $\mathrm{H}_{2} \mathrm{O}_{2}$ in $P$. viticola-inoculated and mock-inoculated leaves

The inoculated leaves were cut into small pieces approximately $1 \mathrm{~cm}^{2}$ in area and were then immersed in a solution containing $1 \mathrm{mg} / \mathrm{mL}$ DAB dissolved in $\mathrm{HCl}$ acidified ( $\mathrm{pH}$ 3.8) distilled water. Leaves were incubated for $8 \mathrm{~h}$ to absorb $\mathrm{DAB}$ and react with $\mathrm{H}_{2} \mathrm{O}_{2}$ and peroxidase. Disks with a diameter of $10 \mathrm{~mm}$ were removed from the inoculated leaf centers, and the disks were fixed and decolorized in a solution of ethanol/chloroform (3:1, v/v) containing $0.15 \%(\mathrm{w} / \mathrm{v})$ trichloroacetic acid for 3-5 days. Next, samples of the leaf disks were clarified in saturated chloral hydrate until they became semitransparent, and 


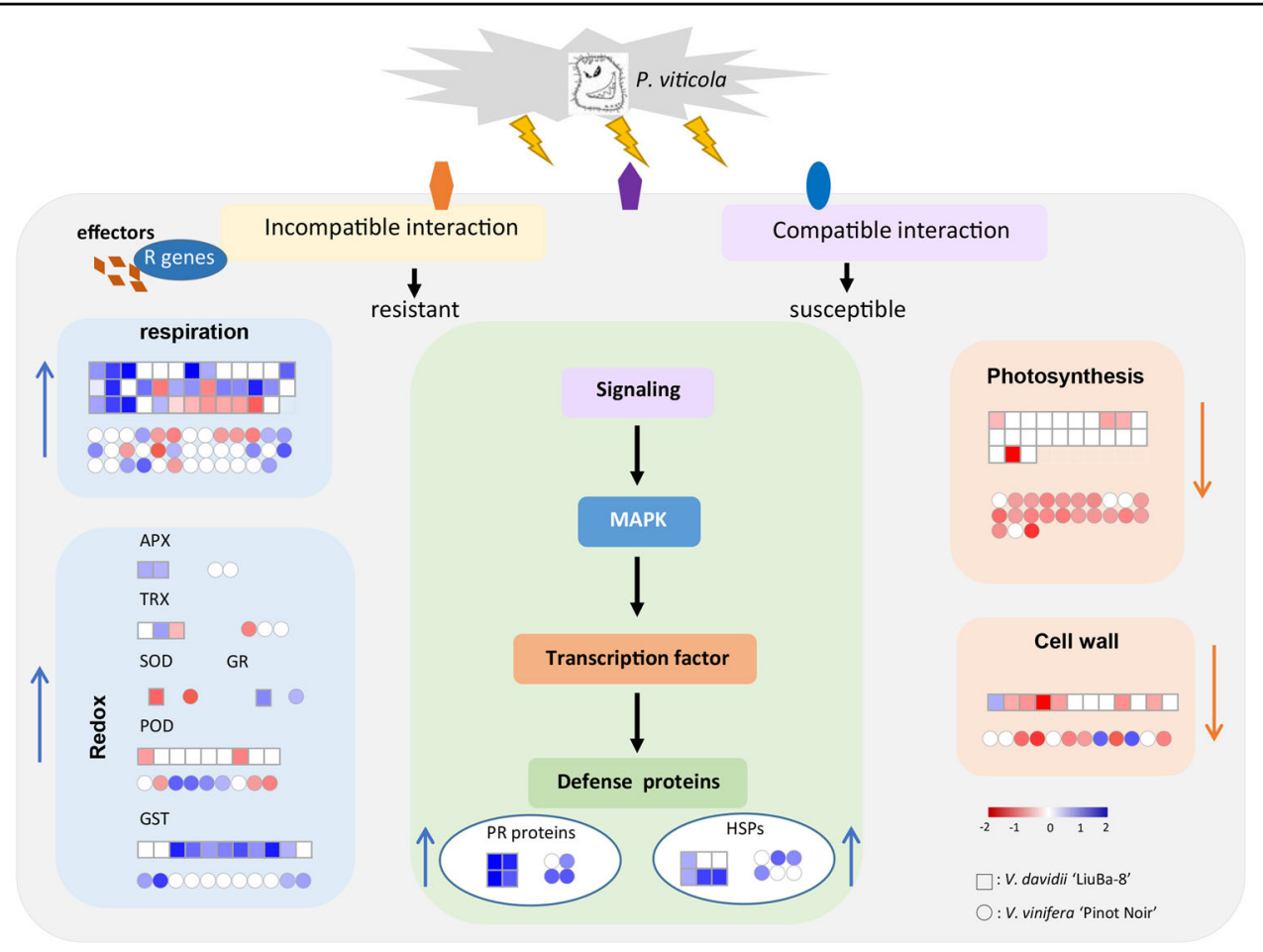

Fig. 9 Schematic overview of DEPs in V. piasezkii "Liuba-8" and V. vinifera "Pinot Noir" at $12 \mathrm{hpi}$

then they were placed in $0.05 \%$ aniline blue $(0.1 \mathrm{M}$ phosphate buffer, $\mathrm{pH}$ 8.0) and incubated at room temperature overnight. The disks were then mounted on microscope slides in the staining solution, with their abaxial surfaces facing upwards. For $P$. viticola, leaf discs were observed under blue-violet light by using fluorescence microscopy. The microscope was an Olympus BX-51, with an excitation wavelength of 400-440 $\mathrm{nm}$ and an emission wavelength of $475 \mathrm{~nm} . \mathrm{H}_{2} \mathrm{O}_{2}$ production was determined by visualization of the samples under bright field conditions and indicated by a reddish-brown coloration.

\section{Sample preparation for iTRAQ}

Total proteins were extracted using the cold-acetone method $^{17}$. Briefly, five volumes of TCA/acetone (1:9) were added to frozen powder of the sample and then mixed vigorously. The mixture was held at $-20^{\circ} \mathrm{C}$ for $4 \mathrm{~h}$ and then centrifuged at $6000 \times g$ for $40 \mathrm{~min}$ at $4{ }^{\circ} \mathrm{C}$. The precipitate was washed with $-20^{\circ} \mathrm{C}$ cold acetone three times and centrifuged at $6000 \times g$ for $40 \mathrm{~min}$ at $4{ }^{\circ} \mathrm{C}$ each time. The solid substance was then air dried, $25 \mathrm{mg}$ of powder was combined with $750 \mu \mathrm{L}$ of SDT buffer, and the mixture was heated to boiling for $5 \mathrm{~min}$. The lysate was then sonicated and heated to boiling for $15 \mathrm{~min}$. The clear liquid on the surface was filtered with a pore size of $0.22 \mu \mathrm{m}$ after centrifugation at $14,000 \times g$ for $40 \mathrm{~min}$, and the protein was quantitated using a BCA Protein Assay Kit. The samples were kept at $-80^{\circ} \mathrm{C}$. For each sample, a medium amount of protein $(20 \mathrm{mg})$ was incorporated into $5 \times$ SDS-PAGE loading buffer, and the mixture was heated to boiling for $5 \mathrm{~min}$. Then, on $12.5 \%$ SDS-PAGE gels, the proteins were separated for 90 minutes by using an invariable current of $14 \mathrm{~mA}$. Proteins were visualized by Coomassie Blue R-250 staining.

\section{Filter-aided sample preparation (FASP digestion) and iTRAQ labeling}

Approximately $200 \mu \mathrm{g}$ of protein from each sample was incorporated into $30 \mu \mathrm{L}$ SDT buffer (4\% SDS, $100 \mathrm{mM}$ DTT, $150 \mathrm{mM}$ Tris-HCl pH 8.0) ${ }^{117}$. UA buffer (8 M urea, $150 \mathrm{mM}$ Tris- $\mathrm{HCl} \mathrm{pH}$ 8.0) was employed to separate DTT, detergent and other LMW elements through repeated ultrafiltration (Microcon units, 10-Kd mass cutoff). Next, to prevent the cysteine residue from being reduced, $100 \mu \mathrm{L}$ of iodoacetamide $(100 \mathrm{mM}$ IAA in UA buffer) was incorporated into samples, which were kept in the dark for $30 \mathrm{~min}$. One hundred microliters of UA buffer was used to clean the filters three times, and then $100 \mu \mathrm{L}$ of DS buffer was used twice. Finally, the protein suspensions were digested with $4 \mu \mathrm{g}$ trypsin (Promega) in $40 \mu \mathrm{L}$ DS buffer overnight at $37^{\circ} \mathrm{C}$, and the resulting peptides were collected as a filtrate. Peptides from each sample were desalted on $\mathrm{C} 18$ cartridges, concentrated by vacuum centrifugation, and reconstituted in $40 \mathrm{Ul} 0.1 \%$ (v/v) formic acid. The peptide content was estimated by UV light spectral density at $280 \mathrm{~nm}$ using an extinction 
coefficient of 1.1 and $0.1 \%(\mathrm{~g} / \mathrm{L})$ solution, which was calculated on the basis of the frequency of tryptophan and tyrosine in vertebrate proteins. The peptide mixture $(100 \mu \mathrm{g})$ from each sample was labeled using iTRAQ reagent according to the manufacturer's instructions.

\section{Peptide fractionation with strong cation exchange (SCX) chromatography}

iTRAQ-labeled peptides were fractionated by SCX chromatography using the AKTA Purifier system (GE Healthcare). The dried peptide mixture was reconstituted and acidified with Buffer A (10 mM KH2PO4 in 25\% ACN, pH 3.0) and loaded onto a PolySULFOETHYL $4.6 \times 100 \mathrm{~mm}$ column. The peptides were sequentially cleaned using different Buffer B $(500 \mathrm{mM} \mathrm{KCl}, 10 \mathrm{mM}$ $\mathrm{KH}_{2} \mathrm{PO}_{4}$ in $25 \% \mathrm{ACN}, \mathrm{pH} 3.0$ ) with a current velocity of 1 milliliter per minute. First, 0-8\% Buffer B was used for the first $22 \mathrm{~min}$, then $8-52 \%$ Buffer B was used from 22 to $47 \mathrm{~min}, 52-100 \%$ Buffer B from 47 to $50 \mathrm{~min}$, and $100 \%$ Buffer B from 50 to $58 \mathrm{~min}$, and finally Buffer B was retuned to $0 \%$ after $58 \mathrm{~min}$. The elution process was supervised by measuring the absorbance at $214 \mathrm{~nm}$, and fractions were generated every minute. C18 cartridges were used to desalt the generated fractions, and then vacuum centrifugation was employed to condense them.

\section{Mass spectrometry (MS)}

The peptide combination was loaded onto a reversedphase trap column connected to a C18 reversed-phase analytical column in Buffer A (0.1\% formic acid) and separated with a linear gradient of Buffer B $(84 \%$ acetonitrile and $0.1 \%$ formic acid) at a flow rate of $300 \mathrm{~nL} / \mathrm{min}$ controlled by IntelliFlow technology. LC-MS/MS analysis was carried out through a Q Exactive mass spectrometer (Thermo Scientific), which functioned in positive ion pattern and was connected to an Easy nLC for $120 \mathrm{~min}$. MS data were generated by adopting a data-dependent top ten approach, which conducts higher energy dissociation fragmentation by picking the rich precursor ions out from the survey scan $(300-1800 \mathrm{~m} / z)$. The target automatic gain control (AGC) was adjusted to $3 \mathrm{e} 6$, the maximum injection duration was $10 \mathrm{~ms}$, and the dynamic exclusion period was $40 \mathrm{~s}$. At $m / z 200$ and at a resolving power of 70,000, survey scans were obtained. The resolving power of the higher energy dissociation spectra was configured to be 17,500 at $\mathrm{m} / z 200$, the normalized energy of collision to be $30 \mathrm{eV}$, the width of the isolation to be $2 \mathrm{~m} / \mathrm{z}$, and the underfill ratio, which is defined as the target value's minimum percentage that may be achieved during the maximum filling period, to be $0.1 \%$. The equipment was operated in activated peptide recognition mode. The MS data have been deposited in the ProteomeXchange Consortium via the PRIDE partner repository with the dataset identifier PXD018845 ${ }^{118}$.

\section{Data analysis}

MASCOT 2.2 was implanted into Proteome Discoverer 1.4 against UniProt Vitis vinifera (20180122, 54701 proteins), and the decoy database was employed to search the MS/MS spectra. The following settings were used for all parameters: trypsin was the digestion enzyme; the maximum missed cleavage was 2; fixed modification: carbamidomethyl (C), iTRAQ 4/8plex (N-term), iTRAQ 4/ 8plex (K); variable modification: oxidation (M), iTRAQ 4/ 8plex (Y); peptide mass tolerance was \pm 20 ppm; fragment mass tolerance was $0.1 \mathrm{Da}$; and the peptide FDR was 0.01 .

\section{Parallel reaction monitoring (PRM) analysis}

To verify the protein expression levels obtained by iTRAQ analysis, four proteins with differential expression levels were further quantified by LC-PRMMS analysis ${ }^{119}$. Briefly, peptides were prepared according to the iTRAQ protocol, and an AQUA stable isotope peptide was spiked in each sample as an internal standard reference. Tryptic peptides were loaded on $\mathrm{C} 18$ stagetips for desalting prior to reversed-phase chromatography on an Easy nLC-1200 system (Thermo Scientific). Liquid chromatography gradients were applied over $1 \mathrm{~h}$ with acetonitrile ranging from 5 to $35 \%$ over $45 \mathrm{~min}$. PRM analysis was conducted through a Q Exactive Plus MS. To employ unique peptides with great confidence and intensity for each targeted protein, an improved collision energy, retention time and charge state for the most notably regulated peptides was obtained through experiments. The MS was performed with positive ion mode, and the parameters were as follows: A complete MS1 scan was achieved at 70,000 resolving power (at $200 \mathrm{~m} / z$ ), the target ACG was $3.0 \times 10^{-6}$, and the maximum ion injection duration was $250 \mathrm{~ms}$. After the complete MS scans, 20 parallel reaction monitoring scans with 35,000 resolution (at $200 \mathrm{~m} / z$ ), AGC value of $3.0 \times 10^{-6}$ and maximum injection duration of $200 \mathrm{~ms}$ were performed. The target peptides were then separated using a 2 Th window. In an HCD collision cell, ions were activated or dissociated at a normalized collision energy of 27. The Skyline method (MacCoss Lab, University of Washington) was used to analyze the raw data ${ }^{120}$. In this analysis, the signal intensities of each greatly changed protein's single peptide sequences were quantified and normalized to a standard reference. The MS proteomics data have been submitted to the ProteomeXchange Consortium through the PRIDE partner repository ${ }^{118}$ with the dataset identifier PXD018868.

\section{Vector construction and transient transformation in $\mathbf{N}$. benthamiana}

Vectors were constructed using the ClonExpress II One Step Cloning Kit (Vazyme Biotech Co., Ltd.) according to the manufacturer's instructions. First, the full-length CDS without the termination codon was amplified from LB and 
PN cDNA using high-fidelity DNA polymerase (KODplus, TOYOBO) and gene-specific primers (Table S5). The primers contained a pair of homologous arms and BamH1 and Sal1 cutting sites at the end of the forward and reverse homologous arms, respectively. The binary vector pCAMBIA2300, which harbors a GFP expression cassette, was digested by the restriction enzymes BamH1 and Sal1. Then, the purified CDS fragments were ligated to the digested vectors by seamless cloning. Finally, the constructed plasmids were introduced into A. tumefaciens GV3101 cells by the freeze-thawing method.

$N$. benthamiana plants were grown in a greenhouse with a 16 -h day at $22^{\circ} \mathrm{C}$ and an 8 -h night at $18{ }^{\circ} \mathrm{C}$. Leaves from 4-to-5-week-old plants were used for transient transformation by agroinfiltration. A. tumefaciens GV3101 carrying binary vectors was cultured in Luria-Bertani (LB) medium with kanamycin at $28^{\circ} \mathrm{C}$ and $180 \mathrm{rpm}$ for $18-24 \mathrm{~h}$. Concentrated Agrobacterium cells were resuspended in infiltration buffer $\left(10 \mathrm{mM} \mathrm{MgCl}_{2}\right.$, $500 \mathrm{mM}$ MES, $200 \mu \mathrm{M}$ acetosyringone) and adjusted to a final OD600 of 0.4-0.6 before infiltration. The suspensions were infiltrated into $N$. benthamiana leaves using a syringe.

\section{Protein extraction and Western blotting}

Agroinfiltrated $N$. benthamiana leaves were harvested at $2 \mathrm{dpi}$ and ground in liquid nitrogen. Total proteins were extracted with PPEB extraction buffer according to our previous study ${ }^{71}$. The extracted proteins were separated using sodium dodecyl sulfate polyacrylamide gel electrophoresis (SDS-PAGE) and transferred to a polyvinylidene fluoride (PVDF) membrane. Mouse monoclonal anti-GFP antibody (Transgen Biotech) diluted at a proportion of 1:4000 in TBST buffer was incubated with PVDF membranes overnight at $4{ }^{\circ} \mathrm{C}$ and washed three times with TBST. Then, goat anti-mouse lgG (H\&L)HRP-conjugated antibody (Jiamay Biotech) was added at a ratio of 1:5000 for $2 \mathrm{~h}$ at room temperature. The PVDF membranes were visualized using an HRP-ECL system to verify whether the protein was expressed.

\section{Pathogen infection assay}

P. capsici strains were routinely grown in V8 agar medium at $28^{\circ} \mathrm{C}$ in the dark. Cultures were maintained in solid-liquid V8 medium for 7 days before zoospores were harvested and used for infection; zoospores were induced by rinsing cultures with sterile water followed by treatment at $4{ }^{\circ} \mathrm{C}$ for $0.5-1 \mathrm{~h}$. Then, $30 \mu \mathrm{L}$ droplets of zoospores were inoculated onto the abaxial side of detached leaves $48 \mathrm{~h}$ post-agroinfiltration, and leaves were incubated for 3-5 days on wet paper towels at $100 \%$ relative humidity. Infections were analyzed by photography. The lesioned $N$. benthamiana leaves were treated in boiling trypan blue solution $(10 \mathrm{~mL}$ lactic acid, $10 \mathrm{~mL}$ glycerol,
$10 \mathrm{~mL} \mathrm{ddH}_{2} \mathrm{O}, 10 \mathrm{~g}$ phenol, $60 \mathrm{~mL}$ absolute ethyl alcohol, and $67 \mathrm{mg}$ trypan blue) for $5 \mathrm{~min}$. Samples were soaked in $2.5 \mathrm{~g} / \mathrm{mL}$ chloral hydrate solution to decolorize and clear the background to observe the area of cell death, which was then recorded.

\section{Acknowledgements}

This work was supported by the National Key Research and Development Program of China (2018YFD1000300, 2019YFD1002500) and National Natural Science Foundation of China (31601716, No. 31972374). We thank Professor Steven van Nocker (MSU Department of Horticulture) for critical and valuable suggestions on the manuscript. We thank Shanghai Applied Protein

Technology Co. Ltd. for help with iTRAQ sequencing and PRM verification.

\section{Author details}

${ }^{1}$ State Key Laboratory of Crop Stress Biology for Arid Areas, College of Horticulture, Northwest A\&F University, Yangling, China. ${ }^{2}$ Key Laboratory of Horticultural Plant Biology and Germplasm Innovation in Northwest China, Ministry of Agriculture, Northwest A\&F University, Yangling, China. ${ }^{3}$ UMR1287 EGFV, CNRS, Université de Bordeaux, INRAE, Bordeaux Sciences Agro, ISW, Villenave d'Ornon, France. ${ }^{4}$ Institute of Botany, The Chinese Academy of Sciences, Beijing, China

\section{Author contributions}

Yan X. and Guo-Tian L. designed the research, Bian-Bian W. conducted the pathogen infection assay, and Bian-Bian W., Mei-Jie L., Ming-Bo L., and Bo-Xing S. prepared the experimental materials for the research. Rui-Qi L. and Xiao Y. conducted the microscopic observations. Guo-Tian L. and Yan X. analyzed the data and wrote the article. Fatma L., Li-Jun W. and David L. revised the manuscript. All authors read and approved the final manuscript.

\section{Conflict of interest}

The authors declare no competing interests.

Supplementary information The online version contains supplementary material available at https://doi.org/10.1038/s41438-021-00533-y.

Received: 15 June 2020 Revised: 16 January 2021 Accepted: 24 February 2021

Published online: 01 May 2021

\section{References}

1. Pieterse, C. M. \& Dicke, M. Plant interactions with microbes and insects: from molecular mechanisms to ecology. Trends Plant Sci. 12, 564-569 (2007).

2. Wang, W., Feng, B.-M., Zhou, J.-M. \& Tang, D.-Z. Plant immune signaling: advancing on two frontiers. J. Integr. plant Biol. 62, 2-24 (2020).

3. Jones, J. D. \& Dangl, J. L. The plant immune system. Nature 444, 323-329 (2006).

4. Jones, J. D. Putting knowledge of plant disease resistance genes to work. Curr. Opin. Plant Biol. 4, 281-287 (2001).

5. Polesani, M. et al. General and species-specific transcriptional responses to downy mildew infection in a susceptible (Vitis vinifera) and a resistant $(\mathrm{V}$. riparia) grapevine species. BMC Genomics 11, 117 (2010).

6. Li, X.-L. et al. Comparative transcriptome analysis reveals defense-related genes and pathways against downy mildew in Vitis amurensis grapevine. Plant Physiol. Biochem. 95, 1-14 (2015).

7. Maytalman, D. et al. Proteomic analysis of early responsive resistance proteins of wheat (Triticum aestivum) to yellow rust (Puccinia striiformis $\mathrm{f}$. sp. tritici) using ProteomeLab PF2D. Plant Omics 6, 24-35 (2013).

8. Bolwell, G. P. \& Wojtaszek, P. Mechanisms for the generation of reactive oxygen species in plant defence-a broad perspective. Physiol. Mol. Plant Pathol. 51, 347-366 (1997).

9. Dai, G. H., Andary, C., Mondolot-Cosson, L. \& Boubals, D. Histochemical studies on the interaction between three species of grapevine, Vitis vinifera, $\mathrm{V}$. rupestris and V. rotundifolia and the downy mildew fungus, Plasmopara viticola. Physiol. Mol. Plant Pathol. 46, 177-188 (1995). 
10. Kortekamp, A. Expression analysis of defence-related genes in grapevine leaves after inoculation with a host and a non-host pathogen. Plant Physiol. Biochem. 44, 58-67 (2006).

11. Figueiredo, A. et al. Transcriptional and metabolic profiling of grape (Vitis vinifera $\mathrm{L}$.) leaves unravel possible innate resistance against pathogenic fungi. J. Exp. Bot. 59, 3371-3381 (2008).

12. Wu, J. et al. Whole genome wide expression profiles of Vitis amurensis grape responding to downy mildew by using Solexa sequencing technology. BMC Plant Biol. 10, 234 (2010).

13. Malacarne, G. et al. Resistance to Plasmopara viticola in a grapevine segregating population is associated with stilbenoid accumulation and with specific host transcriptional responses. BMC Plant Biol. 11, 114 (2011)

14. Liu, R.-Q. et al. Transcriptomic analysis of Chinese wild Vitis pseudoreticulata in response to Plasmopara viticola. Protoplasma 256, 1409-1424 (2019).

15. Fröbel, S., Dudenhöffer, J., Töpfer, R. \& Zyprian, E. Transcriptome analysis of early downy mildew (Plasmopara viticola) defense in grapevines carnying the Asian resistance locus Rpv10. Euphytica 215, 28 (2019).

16. Toffolatti, S. L. et al. Novel aspects on the interaction between grapevine and plasmopara viticola: dual-RNA-seq analysis highlights gene expression dynamics in the pathogen and the plant during the battle for infection. Genes 11, 261 (2020).

17. JorrinNovo, J., Komatsu, S., Weckwerth, W. \& Wienkoop, S. Plant Proteomics: Methods and Protocols (Springer, 2014).

18. Xu, P.-I. et al. Proteomic analysis of the resistant responses of two vitis amurensis cultivars to plasmopara viticola infections. Curr. Proteom. 12, 63-68 (2015).

19. Lemaitre-Guillier, C. et al. Proteomics towards the understanding of elicitor induced resistance of grapevine against downy mildew. J. Proteom. 156, 113-125 (2017).

20. Milli, A. et al. Proteomic analysis of the compatible interaction between Vitis vinifera and Plasmopara viticola. J. Proteom. 75, 1284-1302 (2012).

21. Nascimento-Gavioli, M. C. et al. Proteome of Plasmopara viticola-infected Vitis vinifera provides insights into grapevine Rpv1/Rpv3 pyramided resistance to downy mildew. J. Proteom. 151, 264-274 (2017).

22. Buonassisi, D. et al. Breeding for grapevine downy mildew resistance: a review of "omics" approaches. Euphytica 213, 103 (2017).

23. Figueiredo, A. et al. Specific adjustments in grapevine leaf proteome discriminating resistant and susceptible grapevine genotypes to Plasmopara viticola. J. Proteom. 152, 48-57 (2017).

24. Zieske, L. R. A perspective on the use of iTRAQ reagent technology for protein complex and profiling studies. J. Exp. Bot. 57, 1501-1508 (2006).

25. Liu, R. Q. et al. Histological responses to downy mildew in resistant and susceptible grapevines. Protoplasma 252, 259-270 (2015).

26. Yin, X. et al. Pathogen development and host responses to Plasmopara viticola in resistant and susceptible grapevines: an ultrastructural study. Hortic Res. 4, 17033 (2017).

27. Kortekamp, A. \& Zyprian, E. Characterization of Plasmopara-resistance in grapevine using in vitro plants. J. Plant Physiol. 160, 1393-1400 (2003).

28. Díez-Navajas, A. M., Wiedemann-Merdinoglu, S., Greif, C. \& Merdinoglu, D. Nonhost versus host resistance to the grapevine downy mildew, Plasmopara viticola, studied at the tissue level. Phytopathology 98, 776-780 (2008).

29. Usadel, B. et al. Extension of the visualization tool MapMan to allow statistical analysis of arrays, display of corresponding genes, and comparison with known responses. Plant Physiol. 138, 1195-1204 (2005).

30. Bourmaud, A., Gallien, S. \& Domon, B. Parallel reaction monitoring using quadrupole-orbitrap mass spectrometer: principle and applications. Proteomics 16, 2146-2159 (2016).

31. Bilgin, D. D. et al. Biotic stress globally downregulates photosynthesis genes Plant Cell Environ. 33, 1597-1613 (2010).

32. Liu, G.-T. et al. Differential proteomic analysis of grapevine leavesby iTRAQ reveals responses to heat stress andsubsequent recovery. BMC Plant Biol. 110, 1-17 (2014)

33. Al-Yasi, H. et al. Impact of drought on growth, photosynthesis, osmotic adjustment, and cell wall elasticity in Damask rose. Plant Physiol. Biochem. 150, 133-139 (2020).

34. Scharte, J., Schon, H. \& Weis, E. Photosynthesis and carbohydrate metabolism in tobacco leaves during an incompatible interaction with Phytophthora nicotianae. J. Exp. Bot. 28, 1421-1435 (2005).

35. Bonfig, K. B., Schreiber, U., Gabler, A., Roitsch, T. \& Berger, S. Infection with virulent and avirulent $P$. syringae strains differentially affects photosynthesis and sink metabolism in Arabidopsis leaves. Planta 225, 1-12 (2006).
36. Swarbrick, P. J., Schulze-Lefert, P. \& Scholes, J. D. Metabolic consequences of susceptibility and resistance (race-specific and broad-spectrum) in barley leaves challenged with powdery mildew. Plant Cell Environ. 29, 1061-1076 (2006).

37. Souza, P. F. N., Garcia-Ruiz, H. \& Carvalho, F. E. L. What proteomics can reveal about plant-virus interactions? Photosynthesis-related proteins on the spotlight. Theor. Exp. Plant Physiol. 31, 227-248 (2019).

38. Aro, E.-M., McCaffery, S. \& Anderson, J. M. Photoinhibition and D1 protein degradation in peas acclimated to different growth irradiances. Plant Physiol. 103, 835-843 (1993).

39. Souza, P. F. et al. Photosynthetic and biochemical mechanisms of an EMSmutagenized cowpea associated with its resistance to cowpea severe mosaic virus. Plant Cell Rep. 36, 219-234 (2017).

40. Kundu, S., Chakraborty, D., Kundu, A. \& Pal, A. Proteomics approach combined with biochemical attributes to elucidate compatible and incompatible plant-virus interactions between Vigna mungo and Mungbean Yellow Mosaic India Virus. Proteome Sci. 11, 15 (2013).

41. Abbink, T. E. et al. Silencing of a gene encoding a protein component of the oxygen-evolving complex of photosystem II enhances virus replication in plants. Virology 295, 307-319 (2002)

42. Gnanasekaran, P., Ponnusamy, K. \& Chakraborty, S. A geminivirus betasatellite encoded $B C 1$ protein interacts with PsbP and subverts PsbP-mediated antiviral defence in plants. Mol. Plant Pathol. 20, 943-960 (2019).

43. Mahmood, T., Jan, A. \& Komatsu, S. Proteomic analysis of bacterial blight defence signalling pathway using transgenic rice overexpressing thaumatinlike protein. Biol. Plant. 53, 285-293 (2009).

44. Carmo-Silva, A. E. \& Salvucci, M. E. The regulatory properties of Rubisco activase differ among species and affect photosynthetic induction during light transitions. Plant Physiol. 161, 1645-1655 (2013).

45. Afroz, A., Khan, M. R., Ahsan, N. \& Komatsu, S. Comparative proteomic analysis of bacterial wilt susceptible and resistant tomato cultivars. Peptides $\mathbf{3 0}$ 1600-1607 (2009).

46. Hu, H., Sheng, L., Zhang, G.-Z., Gu, Q. \& Zheng, K.-F. Influence of bacterial leaf blight on the photosynthetic characteristics of resistant and susceptible rice. J. Phytopathol. 166, 547-554 (2018).

47. Debona, D. et al. Limitations to photosynthesis in leaves of wheat plants infected by Pyricularia oryzae. Phytopathology 104, 34-39 (2014).

48. Mutuku, J. M. \& Nose, A. Changes in the contents of metabolites and enzyme activities in rice plants responding to Rhizoctonia solani Kuhn infection: activation of glycolysis and connection to phenylpropanoid pathway. Plant Cell Physiol. 53, 1017-1032 (2012).

49. Hancock, J. T. et al. Proteomic identification of glyceraldehyde 3-phosphate dehydrogenase as an inhibitory target of hydrogen peroxide in Arabidopsis. Plant Physiol. Biochem 43, 828-835 (2005).

50. Han, S.-J. et al. Cytoplastic glyceraldehyde-3-phosphate dehydrogenases interact with ATG3 to negatively regulate autophagy and immunity in Nicotiana benthamiana. Plant Cell 27, 1316-1331 (2015).

51. Henry, E., Fung, N., Liu, J., Drakakaki, G. \& Coaker, G. Beyond glycolysis: GAPDHs are multi-functional enzymes involved in regulation of ROS, autophagy, and plant immune responses. PLoS Genet. 11, e1005199 (2015).

52. Zeng, H.-Q. et al. Molecular identification of GAPDHs in cassava highlights the antagonism of MeGAPCs and MeATG8s in plant disease resistance against cassava bacterial blight. Plant Mol. Biol. Rep. 97, 201-214 (2018).

53. Baek, D. et al. Suppression of reactive oxygen species by glyceraldehyde-3phosphate dehydrogenase. Phytochemistry 69, 333-338 (2008).

54. Kim, K. J. et al. Induction of a cytosolic pyruvate kinase 1 gene during the resistance response to Tobacco mosaic virus in Capsicum annuum. Plant Cell Rep. 25, 359-364 (2006).

55. Restrepo, S. et al. Gene profiling of a compatible interaction between Phytophthora infestans and Solanum tuberosum suggests a role for carbonic anhydrase. MPMI 18, 913-922 (2005).

56. Zulak, K. G., Khan, M. F., Alcantara, J., Schriemer, D. C. \& Facchini, P. J. Plant defense responses in opium poppy cell cultures revealed by liquid chromatography-tandem mass spectrometry proteomics. Mol. Cell Proteom. 8, 86-98 (2009).

57. Danson, J., Wasano, K. \& Nose, A. Infection of rice plants with the sheath blight fungus causes an activation of pentose phosphate and glycolytic pathways. Eur. J. Plant Pathol. 106, 555-561 (2000).

58. Tadege, M. et al. Activation of plant defense responses and sugar efflux by expression of pyruvate decarboxylase in potato leaves. Plant J. 16, 661-671 (1998). 
59. Underwood, W. The plant cell wall: a dynamic barrier against pathogen invasion. Front. Plant Sci. 3, 85 (2012).

60. Maris, A., Suslov, D., Fry, S. C., Verbelen, J. P. \& Vissenberg, K. Enzymic characterization of two recombinant xyloglucan endotransglucosylase/hydrolase (XTH) proteins of Arabidopsis and their effect on root growth and cell wall extension. J. Exp. Bot. 60, 3959-3972 (2009).

61. Han, Y. et al. DkXTH8, a novel xyloglucan endotransglucosylase/hydrolase in persimmon, alters cell wall structure and promotes leaf senescence and fruit postharvest softening. Sci. Rep. 6, 39155 (2016).

62. Herbers, K., Lorences, E. P., Barrachina, C. \& Sonnewald, U. Functional characterisation of Nicotiana tabacum xyloglucan endotransglycosylase (NtXET1): generation of transgenic tobacco plants and changes in cell wall xyloglucan. Planta 212, 279-287 (2001).

63. Alexandersson, E. et al. Constitutive expression of a grapevine polygalacturonase-inhibiting protein affects gene expression and cell wall properties in uninfected tobacco. BMC Res. Notes 4, 1-17 (2015).

64. Yang, L. et al. Expression of a wild eggplant ribosomal protein L13a in potato enhances resistance to Verticillium dahliae. Plant Cell Tissue Org. 115, 329-340 (2013).

65. Gong, Q. et al. Salicylic acid-related cotton (Gossypium arboreum) ribosomal protein GaRPL18 contributes to resistance to Verticillium dahliae. BMC Plant Biol. 17, 59 (2017).

66. $\mathrm{Xu}, \mathrm{X}$-W. et al. Comparative proteomic analysis of cucumber powdery mildew resistance between a single-segment substitution line and its recurrent parent. Hortic. Res. 6, 115 (2019).

67. Hu, X.-B., Song, F.-M. \& Zheng, Z. Molecular characterization and expression analysis of a rice protein phosphatase $2 \mathrm{C}$ gene, OsBIPP2C1, and overexpression in transgenic tobacco conferred enhanced disease resistance and abiotic tolerance. Physiol. Plant. 127, 225-236 (2006).

68. $\mathrm{Hu}, \mathrm{X}$-B. et al. Ectopic expression of a rice protein phosphatase $2 \mathrm{C}$ gene OsBIPP2C2 in tobacco improves disease resistance. Plant Cell Rep. $\mathbf{2 8}$ 985-995 (2009).

69. Christensen, A. B. et al. The molecular characterization of two barley proteins establishes the novel PR-17 family of pathogenesis-related proteins. Mol. Plant Pathol. 3, 135-144 (2002).

70. Dai, L.-M. et al. The novel gene VpPR4-1 from vitis pseudoreticulata increases powdery mildew resistance in transgenic vitis vinifera L. Front. Plant Sci. $\mathbf{7}$, 695 (2016).

71. Li, M.-Y. et al. CRISPR/Cas9-mediated VvPR4b editing decreases downy mildew resistance in grapevine (Vitis vinifera L.). Hortic. Res. 7, 149 (2020).

72. Rajam, M. V. et al. Thaumatin gene confers resistance to fungal pathogens as well as tolerance to abiotic stresses in transgenic tobacco plants. Biol. Plant. 51, 135-141 (2007)

73. Velazhahan, R. \& Muthukrishnan, S. Transgenic tobacco plants constitutively overexpressing a rice thaumatin-like protein (PR-5) show enhanced resistance to Alternaria alternata. Biol. Plant. 47, 347-354 (2003).

74. Schestibratov, K. A. \& Dolgov, S. V. Transgenic strawberry plants expressing a thaumatin II gene demonstrate enhanced resistance to Botrytis cinerea. Sci. Hortic. 106, 177-189 (2005).

75. Monteiro, S., Barakat, M., Piçarra-Pereira, M. A., Teixeira, A. R. \& Ferreira, R. B. Osmotin and thaumatin from grape: a putative general defense mechanism against pathogenic fungi. Phytopathology 93, 1505-1512 (2003).

76. Somssich, I. E., Schmelzer, E., Bollmann, J. \& Hahlbrock, K. Rapid activation by fungal elicitor of genes encoding "pathogenesis-related" proteins in cultured parsley cells. Proc. Natl Acad. Sci. USA 83, 2427-2430 (1986).

77. Zhou, X.-J., Lu, S., Xu, Y.-H., Wang, J.-W. \& Chen, X.-Y. A cotton cDNA (GaPR-10) encoding a pathogenesis-related 10 protein with in vitro ribonuclease activity. Plant Sci. 162, 629-636 (2002).

78. Kim, S. T. et al. The rice pathogen-related protein 10 (JIOsPR10) is induced by abiotic and biotic stresses and exhibits ribonuclease activity. Plant Cell Rep. 27, 593-603 (2008)

79. He, M.-Y. et al. Subcellular localization and functional analyses of a PR10 protein gene from Vitis pseudoreticulata in response to Plasmopara viticola infection. Protoplasma 250, 129-140 (2013).

80. $\mathrm{Su}, \mathrm{H}$. et al. Overexpression of VpPR10.1 by an efficient transformation method enhances downy mildew resistance in V. vinifera. Plant Cell Rep. 37, 819-832 (2018).

81. Ma, H. et al. Grapevine VpPR10.1 functions in resistance to Plasmopara viticola through triggering a cell death-like defence response by interacting with VpVDAC3. Plant Biotechnol. J. 16, 1488-1501 (2018).
82. Park, C.-J. \& Seo, Y.-S. Heat shock proteins: a review of the molecular chaperones for plant immunity. Plant Pathol. J. 31, 323-333 (2015).

83. Liu, J. et al. The Hsp70 gene family in Solanum tuberosum: genome-wide identification, phylogeny, and expression patterns. Sci. Rep. 8, 16628 (2018).

84. Kallamadi, P. R. et al. An Insight into powdery mildew-infected, susceptible, resistant, and immune sunflower genotypes. Proteomics 18, e1700418 (2018).

85. Jacob, P., Hirt, H. \& Bendahmane, A. The heat-shock protein/chaperone network and multiple stress resistance. Plant Biotechnol. J. 15, 405-414 (2017).

86. Szajko, K., Plich, J., Przetakiewicz, J., Soltys-Kalina, D. \& Marczewski, W. Comparative proteomic analysis of resistant and susceptible potato cultivars during Synchytrium endobioticum infestation. Planta 251, 4 (2019).

87. Jelenska, J., van Hal, J. A. \& Greenberg, J. T. Pseudomonas syringae hijacks plant stress chaperone machinery for virulence. Proc. Natl Acad. Sci. USA 107 13177-13182 (2010).

88. Noel, L. D. et al. Interaction between SGT1 and cytosolic/nuclear HSC70 chaperones regulates Arabidopsis immune responses. Plant Cell 19, 4061-4076 (2007).

89. Qi, Y.-C. et al. Over-expression of mitochondrial heat shock protein 70 suppresses programmed cell death in rice. FEBS Lett. 585, 231-239 (2011).

90. Kim, N. H. \& Hwang, B. K. Pepper heat shock protein 70a interacts with the type III effector AvrBsT and triggers plant cell death and immunity. Plant Physiol. 167, 307-322 (2015).

91. Takahashi, A., Casais, C., Ichimura, K. \& Shirasu, K. HSP90 interacts with RAR1 and SGT1 and is essential for RPS2-mediated disease resistance in Arabidopsis. Proc. Natl Acad. Sci. USA 100, 11777-11782 (2003).

92. Hubert, D. A. et al. Cytosolic HSP90 associates with and modulates the Arabidopsis RPM1 disease resistance protein. EMBO J. 22, 5679-5689 (2003).

93. Bos, J. I. et al. The C-terminal half of Phytophthora infestans RXLR effector AVR3a is sufficient to trigger R3a-mediated hypersensitivity and suppress INF1-induced cell death in Nicotiana benthamiana. Plant J. 48, 165-176 (2006).

94. Lopes-Caitar, V. S., Silva, S. M. H. \& Marcelino-Guimaraes, F. C. "Plant Small Heat Shock Proteins and Its Interactions with Biotic Stress" in Heat Shock Proteins and Plants, eds A. A. A. Asea, P. Kaur \& S. K. Calderwood (Cham: Spring international publishing). 2, 19-39 (2016).

95. UI Haq, S. et al. Heat shock proteins: dynamic biomolecules to counter plant biotic and abiotic stresses. Int. J. Mol. Sci. https://doi.org/10.3390/ ijms20215321 (2019).

96. Yogendra, K. N. et al. Transcription factor StWRKY1 regulates phenylpropanoid metabolites conferring late blight resistance in potato. J. Exp. Bot. $\mathbf{6 6}$ 7377-7389 (2015).

97. Van Ooijen, G. et al. The small heat shock protein 20 RSI2 interacts with and is required for stability and function of tomato resistance protein I-2. Plant J. 63, 563-572 (2010).

98. Jing, M.-F. \& Wang, Y.-C. Plant pathogens utilize effectors to Hijack the host endoplasmic reticulum as part of their infection strategy. Engineering $\mathbf{6}$, 500-504 (2020).

99. Qiu, Y.-J., Xi, J., Du, L.-Q. \& Poovaiah, B. W. The function of calreticulin in plant immunity. Plant Signal. Behav. 7, 907-910 (2012).

100. Qiu, Y.-J., Xi, J., Du, L.-Q., Roje, S. \& Poovaiah, B. W. A dual regulatory role of Arabidopsis calreticulin-2 in plant innate immunity. Plant J. 69, 489-500 (2012).

101. Torres, M. A., Jones, J. D. \& Dangl, J. L. Reactive oxygen species signaling in response to pathogens. Plant Physiol. 141, 373-378 (2006).

102. Chen, X.-R., Wang, X.-L., Zhang, Z.-G., Wang, Y.-C. \& Zheng, X.-B. Differences in the induction of the oxidative burst in compatible and incompatible interactions of soybean and Phytophthora sojae. Physiol. Mol. Plant Pathol. 73, 16-24 (2008).

103. Mandal, S., Das, R. K. \& Mishra, S. Differential occurrence of oxidative burst and antioxidative mechanism in compatible and incompatible interactions of Solanum lycopersicum and Ralstonia solanacearum. Plant Physiol. Biochem. 49. 117-123 (2011).

104. Nogueira Junior, A. F., Trankner, M., Ribeiro, R. V., von Tiedemann, A. \& Amorim, L. Photosynthetic cost associated with induced defense to plasmopara viticola in grapevine. Front. Plant Sci. 11, 235 (2020).

105. Mittler, R., Vanderauwera, S., Gollery, M. \& Van Breusegem, F. Reactive oxygen gene network of plants. Trends Plant Sci. 9, 490-498 (2004).

106. Mittler, R. ROS are good. Trends Plant Sci. 22, 11-19 (2017).

107. Dadakova, K. et al. Proteome and transcript analysis of Vitis vinifera cell cultures subjected to Botrytis cinerea infection. J. Proteom. 119, 143-153 (2015). 
108. Marsh, E. et al. Changes in protein abundance during powdery mildew infection of leaf tissues of Cabernet Sauvignon grapevine (Vitis vinifera L.). Proteomics 10, 2057-2064 (2010).

109. Spagnolo, A. et al. Physiological changes in green stems of Vitis vinifera L. CV. Chardonnay in response to esca proper and apoplexy revealed by proteomic and transcriptomic analyses. J. Proteome Res. 11, 461-475 (2012).

110. Chivasa, S. et al. Proteomic analysis of differentially expressed proteins in fungal elicitor-treated Arabidopsis cell cultures. J. Exp. Bot. 57, 1553-1562 (2006).

111. Kim, S. T. et al. Proteomic analysis of pathogen-responsive proteins from rice leaves induced by rice blast fungus, Magnaporthe grisea. Proteomics 4, 3569-3578 (2004).

112. Fang, X.P. et al. Proteomic analysis of strawberry leaves infected with Colletotrichum fragariae. J. Proteom. 75, 4074-4090 (2012).

113. Frova, C. The plant glutathione transferase gene family: genomic structure, functions, expression and evolution. Physiol. Plant. 119, 469-479 (2003).

114. Gullner, G., Komives, T., Kiraly, L. \& Schroder, P. Glutathione S-transferase enzymes in plant-pathogen interactions. Front. Plant Sci. 9, 1836 (2018).
115. Wang, J.-M., Liu, H.-Y., Xu, H.-M., Li, M. \& Kang, Z.-S. Analysis of differentia transcriptional profiling in wheat infected by Blumeria graminis f. sp. tritici using GeneChip. Mol. Biol. Rep. 39, 381-387 (2012).

116. Pei, D.-L. et al. Virus-Induced gene silencing of a putative glutathione Stransferase gene compromised Ol-1-mediated resistance against powdery mildew in tomato. Plant Mol. Biol. Rep. 29, 972-978 (2011).

117. Wisniewski, J. R., Zougman, A., Nagaraj, N. \& Mann, M. Universal sample preparation method for proteome analysis. Nat. Methods 6, 359-362 (2009).

118. Perez-Riverol, Y. et al. The PRIDE database and related tools and resources in 2019: improving support for quantification data. Nucleic Acids Res. 47, D442-D450 (2019).

119. Peterson, A. C., Russell, J. D., Bailey, D. J., Westphall, M. S. \& Coon, J. J. Parallel reaction monitoring for high resolution and high mass accuracy quantitative, targeted proteomics. Mol. Cell Proteom. 11, 1475-1488 (2012).

120. MacLean, B. et al. Skyline: an open source document editor for creating and analyzing targeted proteomics experiments. Bioinformatics 26, 966-968 (2010). 